\title{
Geochemical constraints on the origin and tectonic setting of Chargar intrusions in the Alborz orogenic belt, NW Iran
}

\author{
Seyed Hedayatalah Mousavi Motlagh ${ }^{1}$, Majid Ghaderi $^{1, *}$ (D), \\ Mir Ali Asghar Mokhtari ${ }^{2}$ and Narges Yasami ${ }^{1}$ \\ ${ }^{1}$ Department of Economic Geology, Tarbiat Modares University, Tehran 14115-175, Iran. \\ ${ }^{2}$ Department of Geology, Faculty of Sciences, University of Zanjan, Zanjan 45195-313, Iran. \\ *Corresponding author. e-mail: mghaderi@modares.ac.ir
}

MS received 15 March 2019; revised 23 June 2019; accepted 2 July 2019

Chargar area is located in the southern border of Tarom subzone within the Alborz magmatic belt of NW Iran. Two types of intrusions, mainly present in the southern part of the area, have been identified. These bodies intruded into the Karaj Formation volcanic and volcaniclastic rocks. Type-I intrusions occur in the south and include two magmatic bodies: (a) Gabbro-pyroxene quartz monzodiorite-quartz monzodiorite and (b) quartz syenite. Type-II crops out in the west and has a gabbro - gabbro-diorite composition. Geochemically, Chargar intrusive rocks belong to the high-K calc-alkaline to shoshonitic series and are classified as I-type and metaluminous granitoids. These intrusive rocks show insignificant different distribution patterns of trace elements and REEs, but generally they are characterized by highly enriched large ion lithophile elements (e.g., K and $\mathrm{Ba}$ ) and depleted $\mathrm{Nb}$, similar to that of continental arc magmatism. Type-I intrusions and Type-II mafic intrusion were originated from mantle source with amphibole-bearing spinel peridotite composition. These intrusions formed in a continental arc to post-collisional tectonic setting.

Keywords. Geochemistry; continental arc; post-collision; calc-alkaline magmatism; Chargar; Alborz.

\section{Introduction}

Tarom is a magmatic belt, with an NW-SE trend within the Alborz orogenic belt, NW Iran. This belt is characterized by intensive magmatism during the Eocene. Among the volcanic and volcaniclastic rocks, acidic and intermediate rocks are volumetrically dominant, with subordinate basic rocks. These volcano-sedimentary rocks of Eocene age are known as Karaj Formation (KF). Some intrusions have intruded into the volcanic and volcaniclastic units, mainly trending NW-SE.

The Tarom intrusions range in composition from quartz monzo-gabbro to diorite, quartz monzodiorite, Published online: 12 August 2019 monzonite, quartz monzonite, monzogranite, granodiorite, alkali granite, granite and syeno-granite (e.g., Nabatian et al. 2014, 2016; Esmaeli et al. 2015; Mehrabi et al. 2016; Kouhestani et al. 2017, 2018; Yasami et al. 2018). These I-type intrusions have high-K calc-alkaline to shoshonitic affinity and metaluminous characteristics (Irannezhadi et al. 2007; Azizi et al. 2009; Nabatian et al. 2014, 2016; Yasami et al. 2018) and show enrichment in Large Ion Lithophile Elements (LILE) and Light Rare Earth Elements (LREE) and negative anomalies in High Field Strength Elements (HFSE) and depletion in Heavy Rare Earth Elements (HREE) (Nabatian et al. 2014, 2016; Yasami et al. 2018). The Tarom plutons 
show subduction-related arc magmatic signatures (Nabatian et al. 2016).

The contribution of juvenile sources in the petrogenesis of the Tarom intrusions was indicated by isotope evidence. Tarom plutons parental magma derivation from the sub-continental lithospheric mantle source was suggested by geochemical and isotopic data, which were fluids and melts metasomatized from the subducted Neotethyan slab with a minor crustal contribution. Decompression melting in the final stage of Neotethyan subduction through slab rollback or shortly after the cessation of arc magmatism was created by subsequent hot asthenospheric upwelling and lithospheric extension (Nabatian et al. 2016).

Chargar area is located in the southern border of Tarom magmatic belt. There are some small intrusions in the area which are exposed in the southern and western parts of the Chargar $\mathrm{Cu}-\mathrm{Au}$ deposit. In this study, two different types of intrusions were identified in the area: (1) Intrusions in the south of Chargar deposit consisting of gabbro, pyroxene quartz monzodiorite, quartz monzodiorite and quartz syenite (Type-I). (2) The intrusion in the west of the Chargar deposit with gabbro and gabbro-diorite composition (Type-II). The Type-II intrusion is mineralized and hosts disseminated chalcopyrite, pyrite, and galena, while the Type-I intrusions do not contain any mineralization.

These two types of intrusions have not previously been reported or studied. This paper presents petrological and geochemical characteristics of the barren Type-I intrusions and the first-time reported mineralized Type-II mafic intrusion in the Tarom in order to determine their petrogenesis.

\section{Regional setting}

Alborz Mountains are regarded as part of the Tethyan Eurasian magmatic-metallogenic belt. Eocene normal faults have been recognized in the Alborz Mountains (Guest et al. 2006), and stratigraphic evidence of Eocene subsidence in the Alborz Mountains have been explained in connection with Eocene extension (Brunet et al. 2003; Hassanzadeh et al. 2004; Vincent et al. 2005; Guest et al. 2007; Morley et al. 2009). The Eocene Karaj Formation in the Alborz Mountains comprised of volcanic, volcaniclastic, and sedimentary strata. Eocene units were intruded by plutons of mafic to acidic compositions. $\mathrm{A}{ }^{40} \mathrm{Ar} /{ }^{39} \mathrm{Ar}$ age of $37.2 \pm 0.38$
Ma was yielded from biotites in intruded gabbros inside the Karaj Formation in the east-northeast Tehran (Verdel et al. 2011). These late Eocene gabbros show typical arc characteristics, especially noticeable Nb depletions (Verdel et al. 2011), perhaps the single most unique trace element, typical of arc magmatism (Gill 1981).

The Tarom belt is bounded by the Roudbar and Soltanieh faults to the north and south, respectively. Eastern and northwestern parts of the Tarom run to central Alborz and Azerbaijan (Arasbaran) zones, respectively (Castro et al. 2013). Rock units in the Tarom belt mainly consist of Paleogene volcanic rocks including lava flows and pyroclastic rocks (Eocene Karaj Formation) which are intruded by late Eocene intrusive bodies (Castro et al. 2013; Nabatian et al. 2014, 2016). Intrusions are widespread throughout the Tarom subzone forming two sets of elongated NW-SE trending bodies in the north and the south.

Chargar area is located in the southern part of the Tarom magmatic-metallogenic belt within the Alborz orogenic belt (figure 1). This area is made up of volcanic and volcaniclastic rocks of Eocene age (KF) mainly with intermediate to acidic composition. Some small intrusions were noticed in the volcanic and volcaniclastic rocks. These intrusions crop out in the southern (Type-I) and western (Type-II) parts of the Chargar area (figure 2).

The volcanic and volcaniclastic rocks in the Chargar area consist of rhyolitic to basaltic units. Recent work has shown that they have calcalkaline to high-K calc-alkaline and shoshonitic affinity (Mousavi Motlagh and Ghaderi 2019).

\section{Chargar intrusions}

The south Chargar intrusions are represented by gabbro, pyroxene quartz monzodiorite to quartz monzodiorite and quartz syenite (figure $3 \mathrm{a}$ ). The pyroxene quartz monzodiorite intrusion is intruded by quartz syenite and cut by aplitic veins and dikes (figure $3 \mathrm{~b}$ ). The quartz syenite has a thermal effect on the pyroxene quartz monzodiorite. A common feature of the quartz syenite intrusion is its morphology (curvy outcrop), along with brecciation of adjacent host rocks and their invasion by aplitic veins and dikes (figure 3c). Interpenetrating relations include possible diapiric intrusion of the quartz syenite into the pyroxene quartz monzodiorite bodies (figure 3d). The quartz syenite forms a distinct topographically high area relative to 


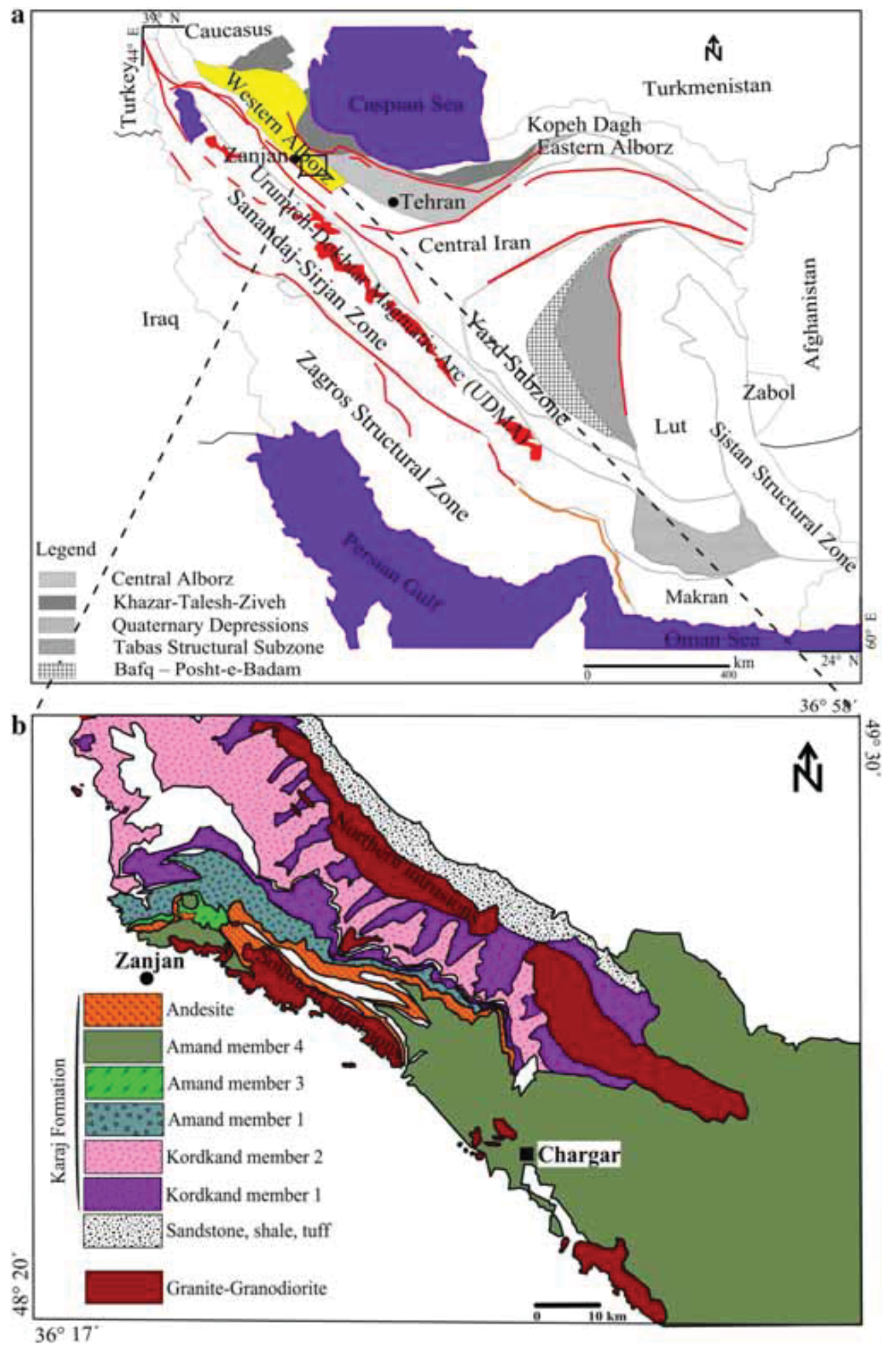

Figure 1. (a) Simplified structural map of Iran, showing the location of the Chargar area within the Cenozoic Alborz magmatic belt; (b) Generalized geological map of the Tarom magmatic belt and location of Chargar Cu-Au deposit. Based on 1:250,000 geological map of Zanjan (modified after Hirayama et al. 1965, 1966).

pyroxene quartz monzodiorite (figure 3e). These field observations suggest that the quartz syenite intrusion is younger than gabbro-pyroxene quartz monzodiorite-quartz monzodiorite.
The Chargar west mineralized intrusion consists of gabbro - gabbro-diorite. This intrusion intruded into the Karaj Formation and has experienced alteration (figure $3 \mathrm{f}$ ). 


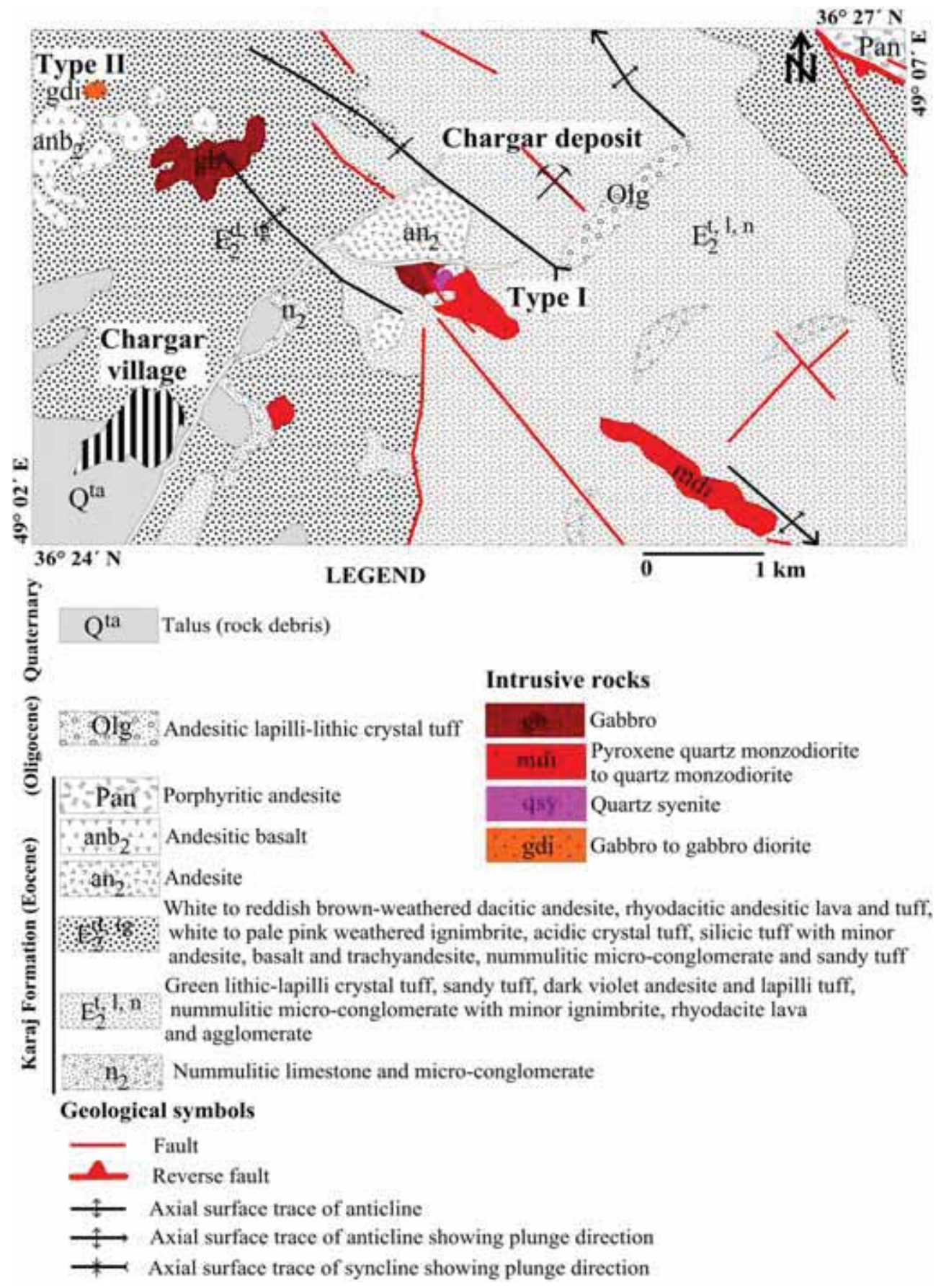

Figure 2. Geological map of the Chargar area, revised and modified based on the new field data from 1:100,000 geological map of Abhar (modified after Hosseini et al. 2017).

\section{Materials and methods}

Fresh and unaltered rocks from Chargar area were collected for petrographic studies and geochemical analysis of major and trace elements using X-Ray Fluorescence (XRF), Inductively Coupled PlasmaOptical Emission Spectrometry (ICP-OES), and Inductively Coupled Plasma-Mass Spectrometry (ICP-MS) techniques. Petrographic studies on 63 microscopic thin sections and XRF analyses on 12 samples were performed at Tarbiat Modares University, Tehran, Iran. Bulk rock major elements of the Chargar samples were analyzed by a Philips PW2404 XRF. It utilizes a rhodium target X-ray tube and a $4 \mathrm{~kW}$ generator to provide the primary X-ray beam. Analytical uncertainties were generally better than $\pm 5 \%$ (2r). Whole-rock major elements were also analyzed by ICP-OES on 

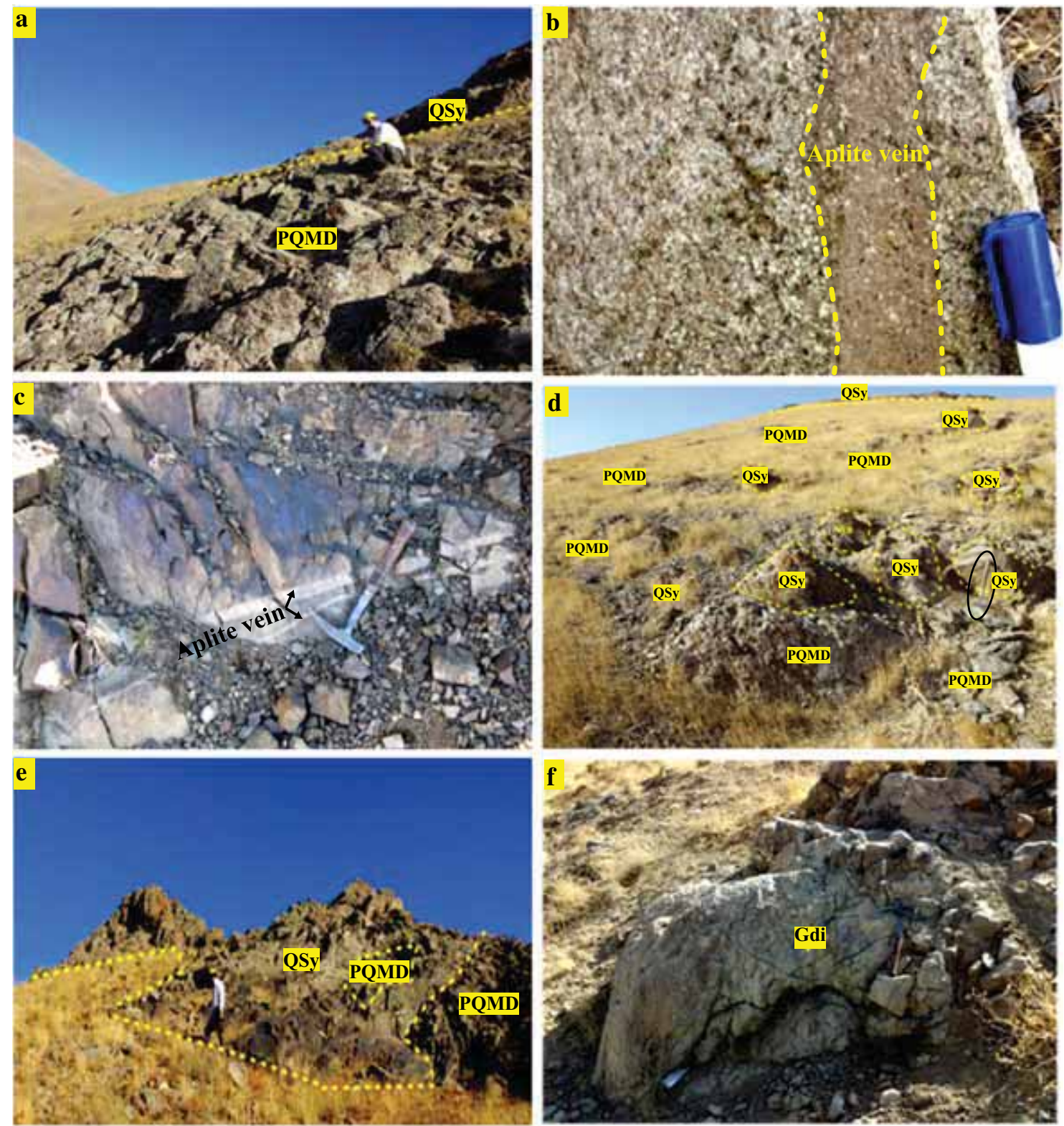

Figure 3. Pictures of the selected studied outcrops of intrusive rocks. (a) Field outcrops of quartz syenite and pyroxene quartz monzodiorite in Chargar area (view to north). (b) Aplite vein cross-cutting the pyroxene quartz monzodiorite. (c) Aplite veins cross-cutting the quartz syenite. (d) The intrusion of the quartz syenite into the pyroxene quartz monzodiorite (view to the northeast). (e) The quartz syenite is topographically higher than pyroxene quartz monzodiorite (view to the north). (f) The Chargar west gabbro - gabbro-diorite intrusion (view to the northeast). QSy: Quartz syenite; PQMD: Pyroxene quartz monzodiorite; Gdi: Gabbro-diorite.

14 samples and trace and rare earth elements by ICP-MS on 25 samples at the Zarazma Company, Iran. The samples were carefully cut into rock chips and were crushed after removal of weathering rinds. Fresh chip samples from different rock types were powdered to 200 meshes using a jaw crusher, hand-picked, and then powdered by low chrome steel disc mill. Then, they preserved for analyses after being dried in an oven at $1000^{\circ} \mathrm{C}$. The major elements were analyzed using ICP-OES after fusion with lithium tetraborate and fluxmeta in a platinum crucible and dissolution by nitric acid, respectively. Analytical accuracy is generally $1 \mathrm{ppb}$ for REE and trace elements. Loss On Ignition (LOI) was determined separately by routine procedures. Powdered samples were dissolved using $\mathrm{HF}, \mathrm{HCl}, \mathrm{HClO}_{4}$ and $\mathrm{HNO}_{3}$ mixture in Teflon beakers for $4 \mathrm{hr}$ at 

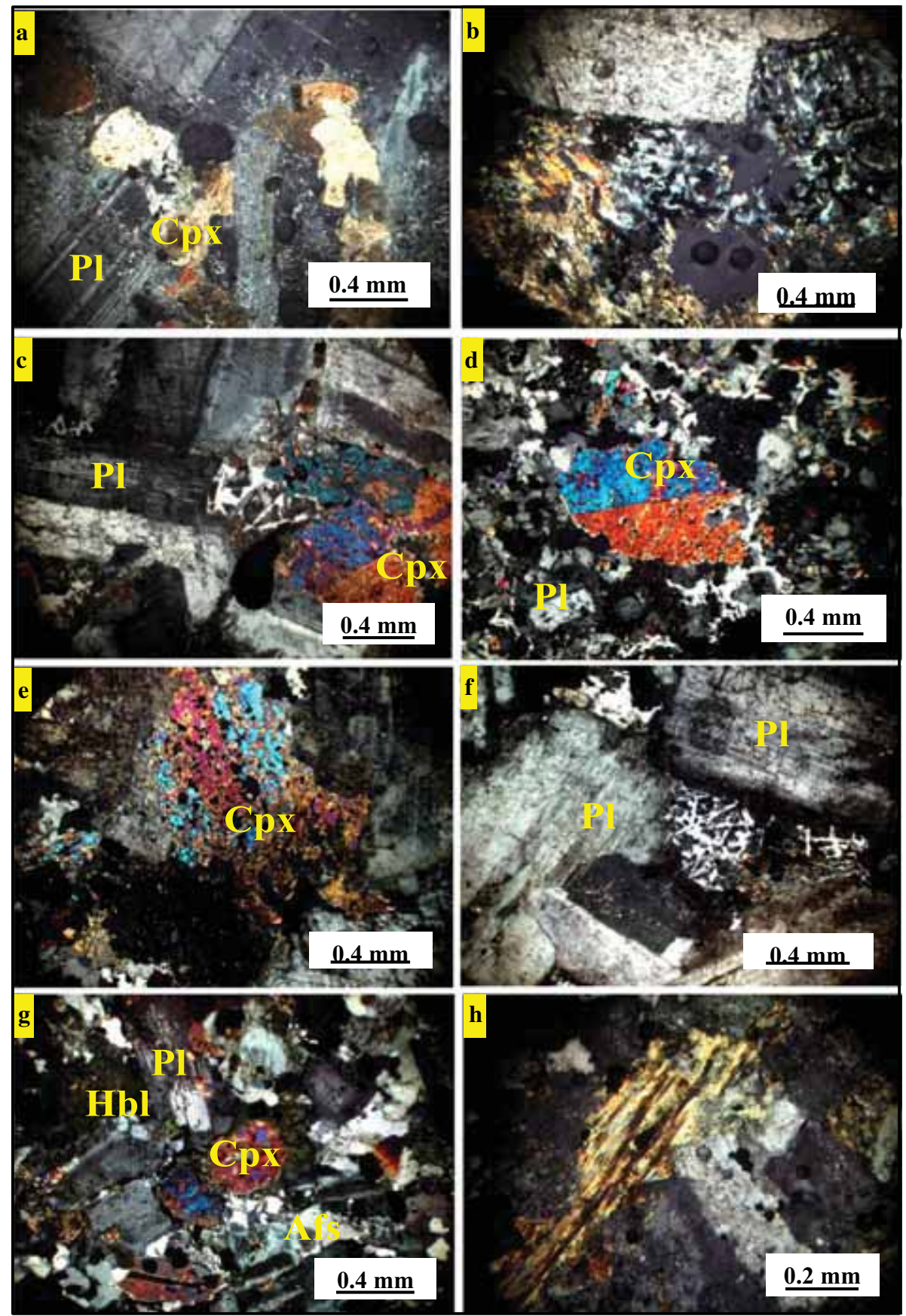

Figure 4. Polarized light microphotographs of gabbro, pyroxene quartz monzodiorite, and quartz monzodiorite. (a) Pyroxene grew between large plagioclase; (b) Pyroxene replaced by chlorite; (c) Pyroxene quartz monzodiorite with granular texture consists of plagioclase and clinopyroxene. Intergrowth of alkali feldspar and quartz can be seen between crystals. (d) Twinned clinopyroxene within fine-grained groundmass in pyroxene quartz monzodiorite. (e) Alteration of clinopyroxene to actinolite and opaque minerals in pyroxene quartz monzodiorite. (f) Quartz and alkali feldspar intergrowth between plagioclase crystals. (g) The granular texture of quartz monzodiorite consists of plagioclase, alkali feldspar, clinopyroxene, and hornblende. Pyroxene crystals replaced by surrounding amphibole. (h) Chlorite and opaque minerals replacing amphibole. Abbreviations from Whitney and Evans (2010). Pl: Plagioclase, Cpx: Clinopyroxene, Hbl: Hornblende, Afs: Alkali feldspar.

$220^{\circ} \mathrm{C}$, followed by dryness at ambient temperature. An internal standard solution was added and the spiked dissolutions were diluted with distilled water. The internal standard solution that is approved by REE standards was utilized for monitoring the signal shift during trace element measurements using an Agilent series 4500 ICP-MS. The precision of the ICP-MS measurements was generally better than $3 \%$ (2r) for most trace elements. 

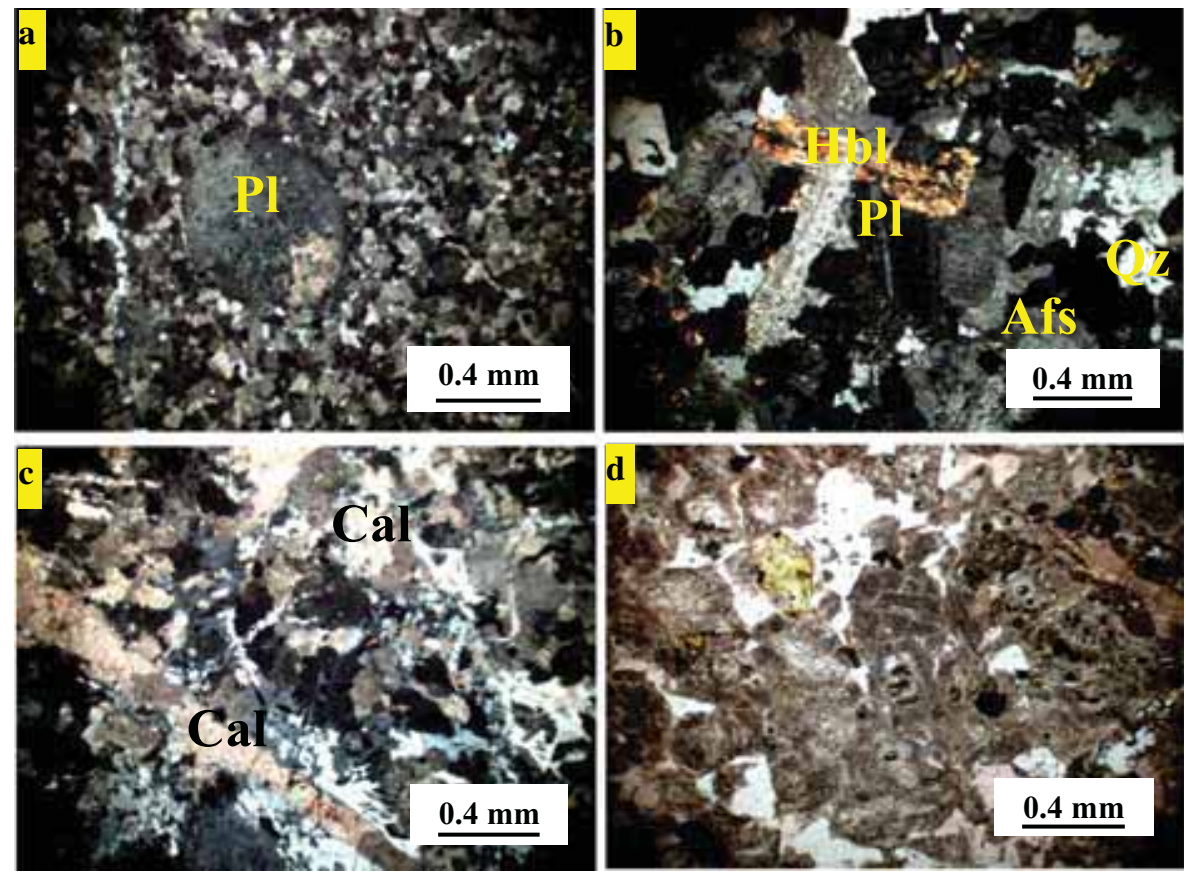

Figure 5. Polarized light microphotographs of quartz syenite. (a) Plagioclase phenocryst in alkali feldspar and quartz groundmass; (b) Alkali feldspar, hornblende, quartz and plagioclase with granular texture; (c) Calcite veinlets crosscutting the rocks; and (d) Argillization of feldspars and chloritization of hornblende. Abbreviations from Whitney and Evans (2010). Pl: Plagioclase, Cal: Calcite, Hbl: Hornblende, Afs: Alkali feldspar, Qz: Quartz.

\section{Results}

\subsection{Petrography}

\subsubsection{Type-I}

The southern intrusions consist of gabbro, pyroxene quartz monzodiorite to quartz monzodiorite and quartz syenite.

Gabbro: The gabbro shows granular and subophitic textures including plagioclase and clinopyroxene. Anhedral-subhedral pyroxene grew between large euhedral and subhedral plagioclase (figure 4a). Coarse-grained plagioclase crystals are replaced by calcite and sericite. Clinopyroxenes have been altered to chlorite (figure 4b). Thin chlorite veinlets cut through the gabbros.

Pyroxene quartz monzodiorite: Pyroxene quartz monzodiorites with granular to porphyritic textures (figure 4c-e) are characterized by significant quantities of euhedral to subhedral twined plagioclase and subhedral to anhedral clinopyroxene. Chlorite, calcite, epidote, sericite, and clay minerals are secondary and form due to alteration of original minerals. Quartz and alkali feldspar are present as small crystals between other minerals. In some places, an intergrowth of alkali feldspar and quartz forms graphic texture (figure 4c, f) which can be related to the late stage of magmatic liquid.

Quartz monzodiorite: The quartz monzodiorite has granular texture (figure $4 \mathrm{~g}$ ) and consists of euhedral to subhedral plagioclase, subhedral to anhedral hornblende, anhedral alkali feldspar, and quartz with a lesser amount of subhedral clinopyroxene and biotite. Accessory minerals are apatite, titanite, magnetite, and zircon. Secondary minerals include calcite, chlorite, sericite, and actinolite. Amphibole is replaced by chlorite and opaque minerals (figure $4 \mathrm{~h}$ ). Some clinopyroxene crystals are replaced by surrounding amphibole (figure $4 \mathrm{~g}$ ). This relationship probably resulted from late hydrous magmatic fluids reacting with pre-existing clinopyroxene to form amphibole.

Quartz syenite: This intrusion has porphyritic to porphyroidic (figure 5a) and granular textures (figure 5b) and is composed of tabular to subhedral alkali feldspar, quartz, euhedral to subhedral plagioclase and euhedral to subhedral hornblende. Plagioclase and alkali feldspar phenocrysts exist in alkali feldspar and quartz groundmass. Clinopyroxene exists as an accessory mineral. Quartz exists as an anhedral mineral in a groundmass of other minerals. The accessory minerals include zircon and apatite. Altered minerals are chlorite, 

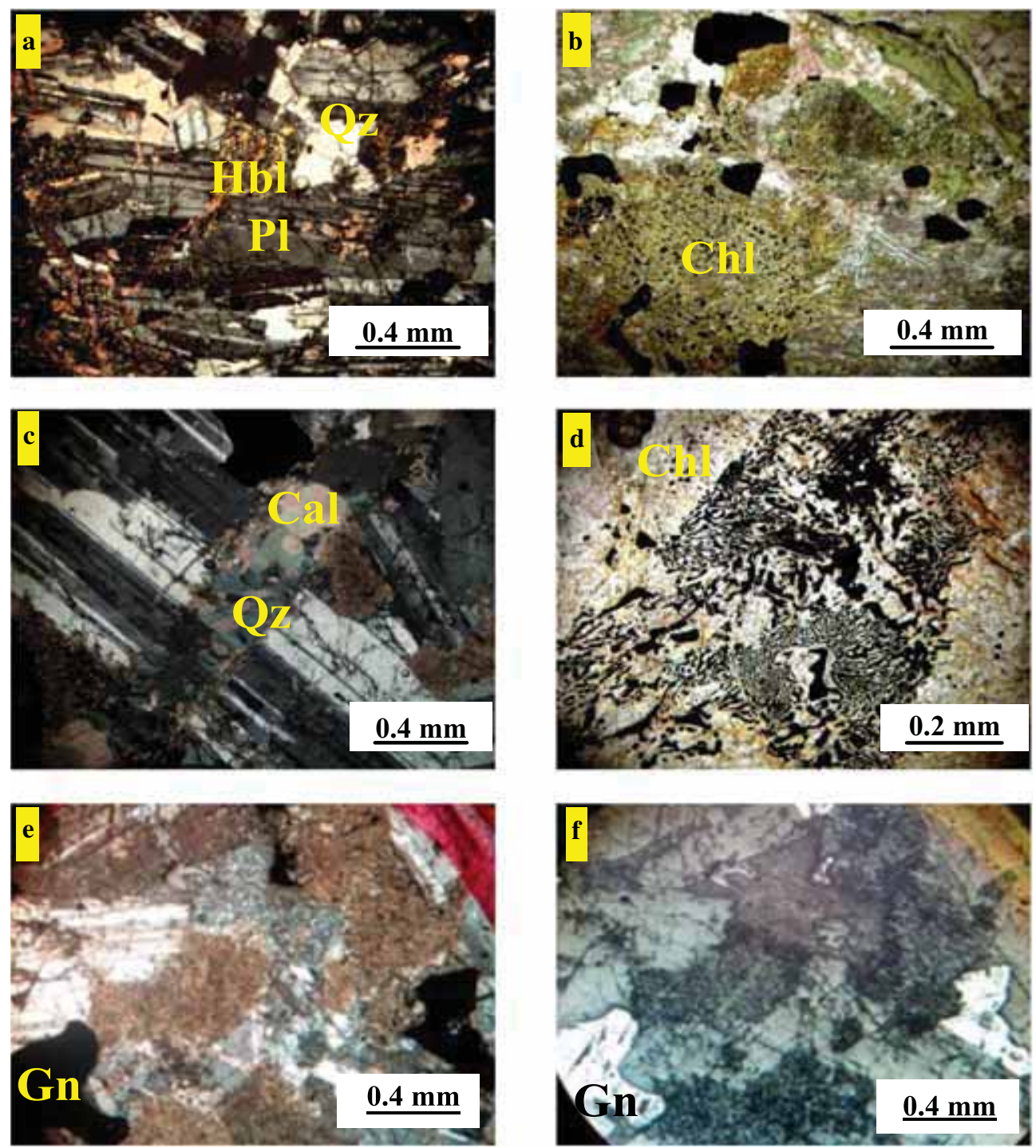

Figure 6. (a-e) Microphotographs of gabbro - gabbro-diorite: (a) The granular texture in gabbro - gabbro-diorite. Plagioclase associated with altered hornblende, quartz and opaque mineral (XPL); (b) Hornblende in gabbro - gabbro-diorite replaced by chlorite and opaque mineral (PPL). (c) Plagioclase crystals cut by quartz-calcite veinlet (XPL); (d) Symplectite texture in which opaque minerals and chlorite replaced ferromagnesian mineral in gabbro - gabbro-diorite (PPL). (e) Sulfide mineralization (opaque minerals) in gabbro - gabbro-diorite unit. (f) Galena mineralization gabbro - gabbro-diorite unit in reflected light. Abbreviations from Whitney and Evans (2010). Pl: Plagioclase, Chl: Chlorite, Cal: Calcite, Hbl: Hornblende, Afs: Alkali feldspar, Qz: Quartz, Gn: Galena.

clay minerals (figure 5d), and calcite. Quartz and calcite veinlets crosscut these rocks (figure $5 \mathrm{c}$ ). Furthermore, calcite is replaced by plagioclase phenocrysts (figure 5c). Most of the alkali feldspar and plagioclase phenocrysts are altered to clay minerals.

\subsubsection{Type-II}

Gabbro and gabbro-diorite: This intrusive rock forms small elongated bodies (figure 3f), dominantly showing granular texture and composed mainly of euhedral to subhedral plagioclase, subhedral amphibole (hornblende) and clinopyroxene (figure 6a). Secondary quartz crystals have grown between magmatic minerals. Chlorite (figure 6b), calcite and clay minerals are secondary minerals. Quartz-calcite veinlets crosscut this intrusion (figure 6c). The symplectite texture can be seen in this unit in which opaque minerals and chlorite replaced ferromagnesian minerals (figure 6d). Most remarkable is the presence of sulfide mineralization as disseminated chalcopyrite, pyrite, galena, and bornite (figure 6e, f) along with some ilmenite and titanomagnetite. 
Table 1. (a) Major oxides in samples from the Chargar intrusions (XRF and ICP-OES). (b) Trace elements of Chargar intrusions $(I C P-M S)$.

(a)

\begin{tabular}{|c|c|c|c|c|c|c|c|c|c|c|c|c|c|}
\hline $\begin{array}{l}\text { Rock } \\
\text { type }\end{array}$ & $\begin{array}{l}\text { Unit } \\
\mathrm{DL}\end{array}$ & $\begin{array}{c}\mathrm{SiO}_{2} \\
\% \\
0.05 \\
\end{array}$ & $\begin{array}{c}\mathrm{Al}_{2} \mathrm{O}_{3} \\
\% \\
0.05 \\
\end{array}$ & $\begin{array}{c}\mathrm{CaO} \\
\% \\
0.05 \\
\end{array}$ & $\begin{array}{c}\mathrm{Fe}_{2} \mathrm{O}_{3} \\
\% \\
0.05 \\
\end{array}$ & $\begin{array}{c}\mathrm{K}_{2} \mathrm{O} \\
\% \\
0.05 \\
\end{array}$ & $\begin{array}{c}\mathrm{MgO} \\
\% \\
0.05 \\
\end{array}$ & $\begin{array}{c}\mathrm{MnO} \\
\% \\
0.05 \\
\end{array}$ & $\begin{array}{c}\mathrm{Na}_{2} \mathrm{O} \\
\% \\
0.05 \\
\end{array}$ & $\begin{array}{c}\mathrm{P}_{2} \mathrm{O}_{5} \\
\% \\
0.05 \\
\end{array}$ & $\begin{array}{c}\mathrm{TiO}_{2} \\
\% \\
0.05 \\
\end{array}$ & $\begin{array}{c}\text { LOI } \\
\% \\
0.05 \\
\end{array}$ & Total \\
\hline PQMD & $\mathrm{H}-2$ & 55.96 & 14.71 & 6.95 & 10.27 & 2.20 & 3.32 & 0.27 & 3.37 & 0.17 & 1.39 & 1.35 & 99.96 \\
\hline PQMD & H-3 & 58.00 & 14.48 & 5.71 & 9.93 & 2.74 & 2.69 & 0.27 & 3.40 & 0.14 & 1.31 & 1.27 & 99.94 \\
\hline QMD & $\mathrm{H}-5$ & 61.43 & 14.76 & 5.30 & 7.45 & 2.96 & 2.45 & 0.17 & 3.58 & 0.11 & 0.98 & 0.77 & 99.96 \\
\hline QMD & H-6 & 56.63 & 16.20 & 8.00 & 7.68 & 2.08 & 2.62 & 0.15 & 4.54 & 0.18 & 1.13 & 0.68 & 99.89 \\
\hline QSy & $\mathrm{H}-7$ & 65.88 & 14.71 & 2.68 & 5.27 & 3.82 & 1.34 & 0.10 & 4.50 & 0.05 & 0.77 & 0.85 & 99.97 \\
\hline QMD & $\mathrm{H}-8$ & 55.82 & 15.98 & 7.23 & 8.57 & 2.14 & 3.29 & 0.22 & 4.28 & 0.10 & 1.16 & 1.17 & 99.96 \\
\hline QSy & $\mathrm{H}-8^{\prime}$ & 64.19 & 14.88 & 3.72 & 5.39 & 3.68 & 1.72 & 0.11 & 4.15 & 0.07 & 0.85 & 1.16 & 99.92 \\
\hline QSy & H-9 & 63.87 & 16.63 & 5.24 & 2.37 & 2.63 & 1.52 & 0.05 & 5.84 & 0.05 & 0.62 & 1.22 & 100.04 \\
\hline QMD & H-11 & 58.89 & 16.12 & 5.04 & 6.60 & 2.76 & 3.11 & 0.17 & 4.59 & 0.05 & 0.96 & 1.62 & 99.91 \\
\hline PQMD & H-12 & 52.59 & 15.35 & 5.93 & 10.17 & 2.94 & 3.20 & 0.46 & 4.91 & 0.14 & 1.37 & 2.72 & 99.78 \\
\hline QMD & H-14 & 58.11 & 15.72 & 8.12 & 5.40 & 2.49 & 3.27 & 0.13 & 4.06 & 0.22 & 1.10 & 1.32 & 99.94 \\
\hline QSy & H-15 & 64.36 & 15.06 & 2.88 & 5.37 & 5.01 & 1.50 & 0.22 & 3.68 & 0.13 & 0.84 & 0.88 & 99.93 \\
\hline PQMD & H-16 & 54.96 & 15.68 & 8.21 & 9.19 & 2.05 & 3.91 & 0.18 & 3.78 & 0.22 & 1.25 & 0.84 & 100.00 \\
\hline PQMD & CH-88 & 55.13 & 15.54 & 7.14 & 8.52 & 2.61 & 3.75 & 0.15 & 3.57 & 0.66 & 1.09 & 0.73 & 98.89 \\
\hline QSy & CH-89 & 62.91 & 14.09 & 9.30 & 1.52 & 3.19 & 0.46 & 0.14 & 5.41 & 0.13 & 0.80 & 1.92 & 99.87 \\
\hline PQMD & CH-90 & 56.60 & 15.58 & 6.77 & 8.82 & 3.16 & 2.99 & 0.17 & 3.93 & 0.48 & 0.89 & 0.52 & 99.91 \\
\hline QMD & CH-91 & 61.80 & 15.01 & 5.22 & 6.54 & 3.57 & 2.59 & 0.11 & 3.18 & 0.42 & 0.91 & 0.53 & 99.88 \\
\hline QSy & CH-97 & 64.86 & 15.51 & 1.90 & 3.57 & 3.69 & 1.43 & 0.07 & 4.85 & 0.19 & 0.59 & 2.21 & 99.85 \\
\hline QSy & CH-101 & 64.56 & 15.82 & 2.58 & 3.27 & 3.81 & 1.99 & 0.10 & 3.62 & 0.21 & 0.57 & 2.67 & 99.20 \\
\hline PQMD & CH-104 & 54.29 & 15.56 & 6.89 & 8.49 & 2.66 & 3.32 & 0.16 & 4.03 & 0.48 & 0.90 & 2.03 & 98.83 \\
\hline PQMD & CH-105 & 54.73 & 15.55 & 6.90 & 7.21 & 3.21 & 3.93 & 0.33 & 4.40 & 0.56 & 1.00 & 1.06 & 98.88 \\
\hline QMD & CH-107 & 58.24 & 15.21 & 4.79 & 8.56 & 2.53 & 3.11 & 0.19 & 3.96 & 1.12 & 1.33 & 0.85 & 99.89 \\
\hline QSy & CH-108* & 64.58 & 15.19 & 2.44 & 5.06 & 3.45 & 2.12 & 0.13 & 4.05 & 0.42 & 0.75 & 0.87 & 99.08 \\
\hline QSy & CH-113 & 65.01 & 15.23 & 2.69 & 4.78 & 3.08 & 2.09 & 0.08 & 3.77 & 0.15 & 0.47 & 2.46 & 99.81 \\
\hline Gdi & CH-117 & 49.93 & 14.86 & 11.42 & 9.40 & 2.76 & 5.13 & 0.10 & 2.86 & 0.15 & 0.95 & 2.35 & 99.91 \\
\hline Gdi & $\mathrm{CH}-117^{*}$ & 50.04 & 15.01 & 11.25 & 9.17 & 2.39 & 5.62 & 0.20 & 2.11 & 0.21 & 0.91 & 2.23 & 99.14 \\
\hline
\end{tabular}

(b)

\begin{tabular}{|c|c|c|c|c|c|c|c|c|c|c|c|c|c|c|c|c|}
\hline $\begin{array}{l}\text { Rock } \\
\text { type } \\
\end{array}$ & $\begin{array}{l}\text { Unit } \\
\text { DL }\end{array}$ & $\begin{array}{c}\text { As } \\
\text { ppm } \\
0.1 \\
\end{array}$ & $\begin{array}{c}\mathrm{Ba} \\
\mathrm{ppm} \\
1 \\
\end{array}$ & $\begin{array}{c}\mathrm{Ce} \\
\mathrm{ppm} \\
0.5 \\
\end{array}$ & $\begin{array}{c}\text { Co } \\
\text { ppm } \\
1 \\
\end{array}$ & $\begin{array}{c}\text { Cs } \\
\text { ppm } \\
0.5 \\
\end{array}$ & $\begin{array}{c}\mathrm{Eu} \\
\mathrm{ppm} \\
0.1 \\
\end{array}$ & $\begin{array}{c}\text { Hf } \\
\text { ppm } \\
0.5 \\
\end{array}$ & $\begin{array}{c}\mathrm{Nb} \\
\mathrm{ppm} \\
1 \\
\end{array}$ & $\begin{array}{c}\mathrm{Pb} \\
\mathrm{ppm} \\
1 \\
\end{array}$ & $\begin{array}{c}\mathrm{Rb} \\
\mathrm{ppm} \\
1 \\
\end{array}$ & $\begin{array}{c}\mathrm{Sr} \\
\mathrm{ppm} \\
1 \\
\end{array}$ & $\begin{array}{c}\text { Th } \\
\text { ppm } \\
0.1\end{array}$ & $\begin{array}{c}\mathrm{Y} \\
\mathrm{ppm} \\
0.5\end{array}$ & $\begin{array}{c}\mathrm{Yb} \\
\mathrm{ppm} \\
0.05\end{array}$ & $\begin{array}{c}\mathrm{Zr} \\
\mathrm{ppm} \\
5\end{array}$ \\
\hline PQMD & $\mathrm{H}-2$ & 3.2 & 700 & 62 & 23.7 & $<0.5$ & 1.14 & 1.52 & 6.7 & 1 & 20 & 337.7 & 3.98 & 32.9 & 4 & 57 \\
\hline PQMD & $\mathrm{H}-3$ & 6 & 3995 & 66 & 19.9 & 0.5 & 1.41 & 1.22 & 7.4 & 12 & 32 & 456.2 & 4.4 & 35.6 & 4.1 & 45 \\
\hline QMD & $\mathrm{H}-5$ & 1.3 & 738 & 67 & 17.2 & 0.6 & 1.05 & 1.31 & 7.3 & 6 & 44 & 292.5 & 5.53 & 33.3 & 3.7 & 40 \\
\hline QMD & H-6 & 2.5 & 618 & 59 & 15.4 & $<0.5$ & 1.15 & 1.16 & 8.3 & 6 & 25 & 488.9 & 4.08 & 31.4 & 3.5 & 43 \\
\hline QSy & $\mathrm{H}-7$ & 3.3 & 1029 & 44 & 9.9 & 0.6 & 0.9 & 0.85 & 8.3 & 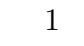 & 48 & 296.2 & 6.7 & 33.2 & 3.2 & 28 \\
\hline QMD & H-8 & 4.1 & 658 & 47 & 21.7 & $<0.5$ & 1.04 & 0.73 & 7.2 & 1 & 28 & 478 & 3.93 & 28 & 3.3 & 27 \\
\hline QSy & H-8 ${ }^{\prime}$ & 5 & 1059 & 79 & 11.4 & 0.6 & 1.04 & 0.89 & 8 & 9 & 44 & 368.4 & 7.11 & 33.4 & 3.4 & 46 \\
\hline QSy & H-9 & 5 & 143 & 44 & 4.8 & $<0.5$ & 0.67 & 1.52 & 8.3 & 1 & $<1$ & 547.5 & 4.39 & 14 & 1.3 & 57 \\
\hline QMD & H-11 & 9.4 & 864 & 64 & 14.2 & 1.4 & 1.14 & 0.73 & 8 & 10 & 2 & 493.4 & 4.33 & 26.5 & 2.9 & 15 \\
\hline PQMD & H-12 & 4.6 & 1396 & 56 & 16.7 & 0.7 & 1.33 & 0.83 & 7.0 & 27 & 16 & 467.4 & 3.45 & 29.6 & 3.6 & 17 \\
\hline QMD & H-14 & 4.9 & 262 & 82 & 11.4 & 0.7 & 1.49 & 3.08 & 8.7 & 3 & 4 & 597.8 & 4.96 & 39 & 4.2 & 138 \\
\hline QSy & H-15 & 4.3 & 937 & 74 & 11.4 & 0.8 & 1.03 & 0.94 & 8.6 & 19 & 93 & 233.9 & 8.12 & 33.3 & 3.6 & 18 \\
\hline PQMD & H-16 & 2 & 537 & 62 & 24 & 0.6 & 1.23 & 2.46 & 8.4 & 1 & 31 & 394.8 & 4.34 & 29.9 & 3.6 & 103 \\
\hline QSy & CH-88 & 3.2 & 605 & 63 & 24 & 1.1 & 1.32 & 1.97 & 6.3 & 1 & 28 & 437.7 & 4.25 & 32.3 & 3.8 & 76 \\
\hline PQMD & CH-89 & 1.4 & 8 & 55 & 1.9 & $<0.5$ & 0.81 & 1.77 & 8.1 & 6 & $<1$ & 142.1 & 5.3 & 24.3 & 2.4 & 54 \\
\hline QMD & CH-90 & 0.5 & 525 & 54 & 22.1 & 0.6 & 1.12 & 1.41 & 9.1 & 1 & 78 & 381.4 & 4.54 & 27.6 & 3.3 & 40 \\
\hline QSy & CH-91 & 2 & 715 & 66 & 17.9 & 0.6 & 1.09 & 1.34 & 9.4 & 4 & 47 & 288.9 & 6.22 & 33.2 & 3.8 & 36 \\
\hline QSy & CH-97 & 0.2 & 764 & 58 & 6.4 & 1.8 & 0.88 & 3.19 & 7.2 & 1 & 16 & 526.5 & 4.48 & 24.2 & 2.7 & 147 \\
\hline
\end{tabular}


Table 1. (Continued.)

(b)

\begin{tabular}{|c|c|c|c|c|c|c|c|c|c|c|c|c|c|c|c|c|}
\hline Rock & Unit & $\begin{array}{c}\text { As } \\
\mathrm{ppm}\end{array}$ & $\begin{array}{c}\mathrm{Ba} \\
\mathrm{ppm}\end{array}$ & $\begin{array}{c}\mathrm{Ce} \\
\mathrm{ppm}\end{array}$ & $\begin{array}{c}\text { Co } \\
\text { ppm }\end{array}$ & $\begin{array}{c}\mathrm{Cs} \\
\mathrm{ppm}\end{array}$ & $\begin{array}{c}\mathrm{Eu} \\
\mathrm{ppm}\end{array}$ & $\begin{array}{c}\text { Hf } \\
\text { ppm }\end{array}$ & $\begin{array}{c}\mathrm{Nb} \\
\mathrm{ppm}\end{array}$ & $\begin{array}{c}\mathrm{Pb} \\
\mathrm{ppm}\end{array}$ & $\begin{array}{c}\mathrm{Rb} \\
\mathrm{ppm}\end{array}$ & $\begin{array}{c}\mathrm{Sr} \\
\mathrm{ppm}\end{array}$ & $\begin{array}{c}\text { Th } \\
\text { ppm }\end{array}$ & $\begin{array}{c}\mathrm{Y} \\
\mathrm{ppm}\end{array}$ & $\begin{array}{c}\mathrm{Yb} \\
\mathrm{ppm}\end{array}$ & $\begin{array}{c}\mathrm{Zr} \\
\mathrm{ppm}\end{array}$ \\
\hline type & DL & 0.1 & 1 & 0.5 & 1 & 0.5 & 0.1 & 0.5 & 1 & 1 & 1 & 1 & 0.1 & 0.5 & 0.05 & 5 \\
\hline PQMD & CH-104 & 7.3 & 509 & 47 & 20.9 & 1.2 & 1.14 & 1.26 & 7.1 & 1 & 37 & 542.3 & 3.04 & 26.2 & 3.2 & 43 \\
\hline PQMD & CH-105 & 3.2 & $>1 \%$ & 56 & 19.3 & $<0.5$ & 2.08 & 1.96 & 9.2 & 15 & 14 & 711.5 & 4.15 & 27.9 & 3.4 & 64 \\
\hline QMD & CH-107 & 4.9 & 729 & 88 & 20.1 & 0.9 & 1.8 & 3.4 & 11.2 & 2 & 25 & 292.7 & 4.84 & 44.6 & 4.9 & 138 \\
\hline $\mathrm{Gb}$ & CH-108 & 0.7 & 349 & 44 & 12 & 1.5 & 1.07 & 1.84 & 6.3 & 15 & 26 & 292.8 & 3.03 & 23.7 & 2.6 & 74 \\
\hline QSy & CH-108* & 4 & 866 & 78 & 12.5 & 0.9 & 1.2 & 1.26 & 9.6 & 5 & 70 & 277.5 & 7.76 & 36 & 3.9 & 31 \\
\hline Gdi & CH-117 & 1.6 & 3762 & 24 & 30.7 & 2.3 & 1 & 1.38 & 5.2 & 3 & $<1$ & 365.5 & 1.71 & 16.4 & 2.8 & 45 \\
\hline Gdi & $\mathrm{CH}-117^{*}$ & 1.7 & 298 & 33 & 28.5 & 0.9 & 0.78 & 1.41 & 5.9 & 7 & 3 & 444.2 & 2.42 & 19.2 & 2.8 & 46 \\
\hline
\end{tabular}

Abbreviations. QSy: Quartz syenite; PQMD: Pyroxene quartz monzodiorite; QMD: Quartz monzodiorite; Gb: Gabbro; Gdi: Gabbro diorite.
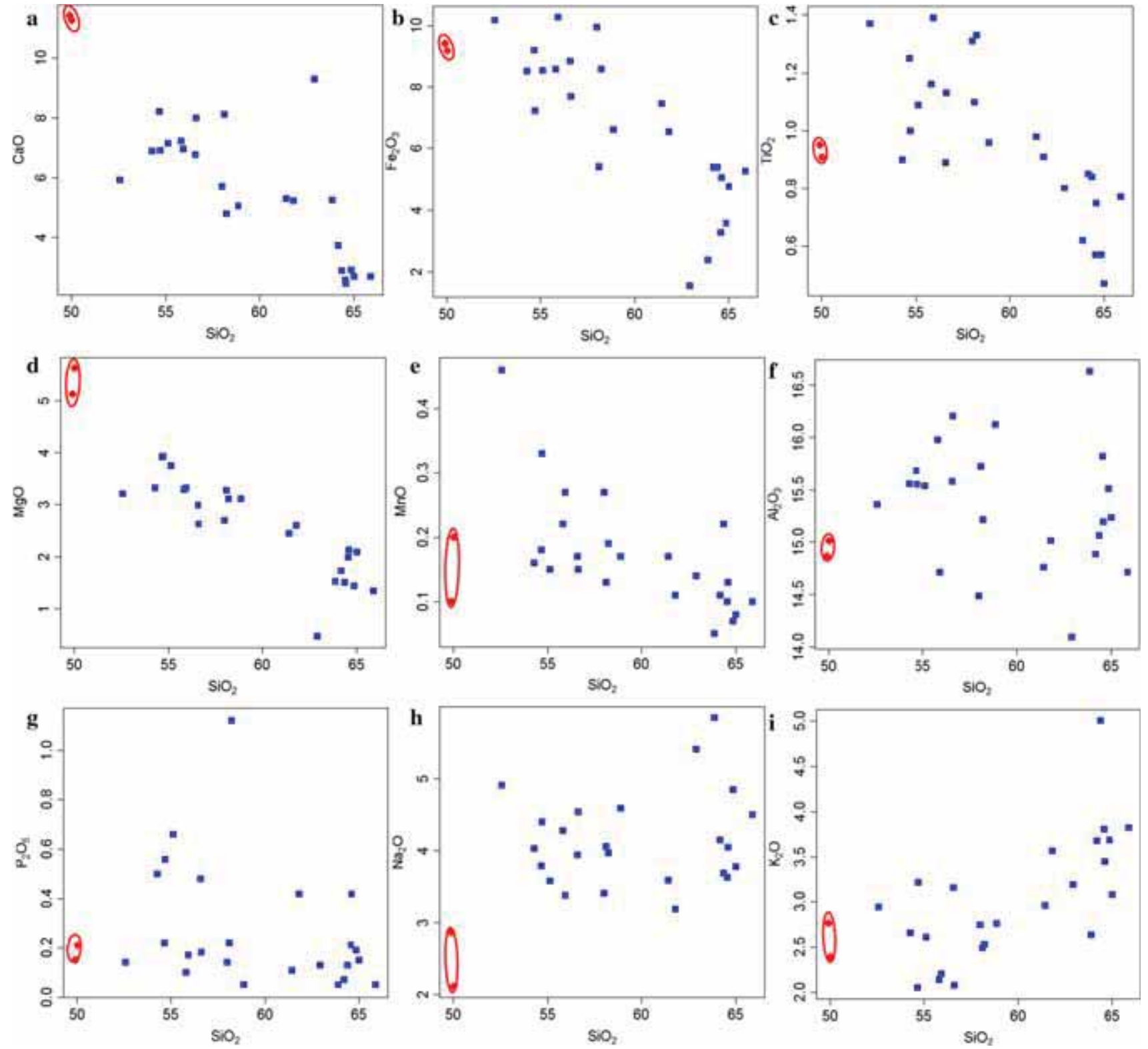

Figure 7. Harker diagrams of $\mathrm{CaO}, \mathrm{Fe}_{2} \mathrm{O}_{3}, \mathrm{TiO}_{2}, \mathrm{MgO}, \mathrm{MnO}, \mathrm{Al}_{2} \mathrm{O}_{3}, \mathrm{P}_{2} \mathrm{O}_{5}, \mathrm{Na}_{2} \mathrm{O}$ and $\mathrm{K}_{2} \mathrm{O}$ vs. $\mathrm{SiO}_{2}$ for the two rock types from Chargar. Blue filled square: southern intrusions (Type-I), red filled circle: western gabbro - gabbro-diorite intrusion (Type-II). 

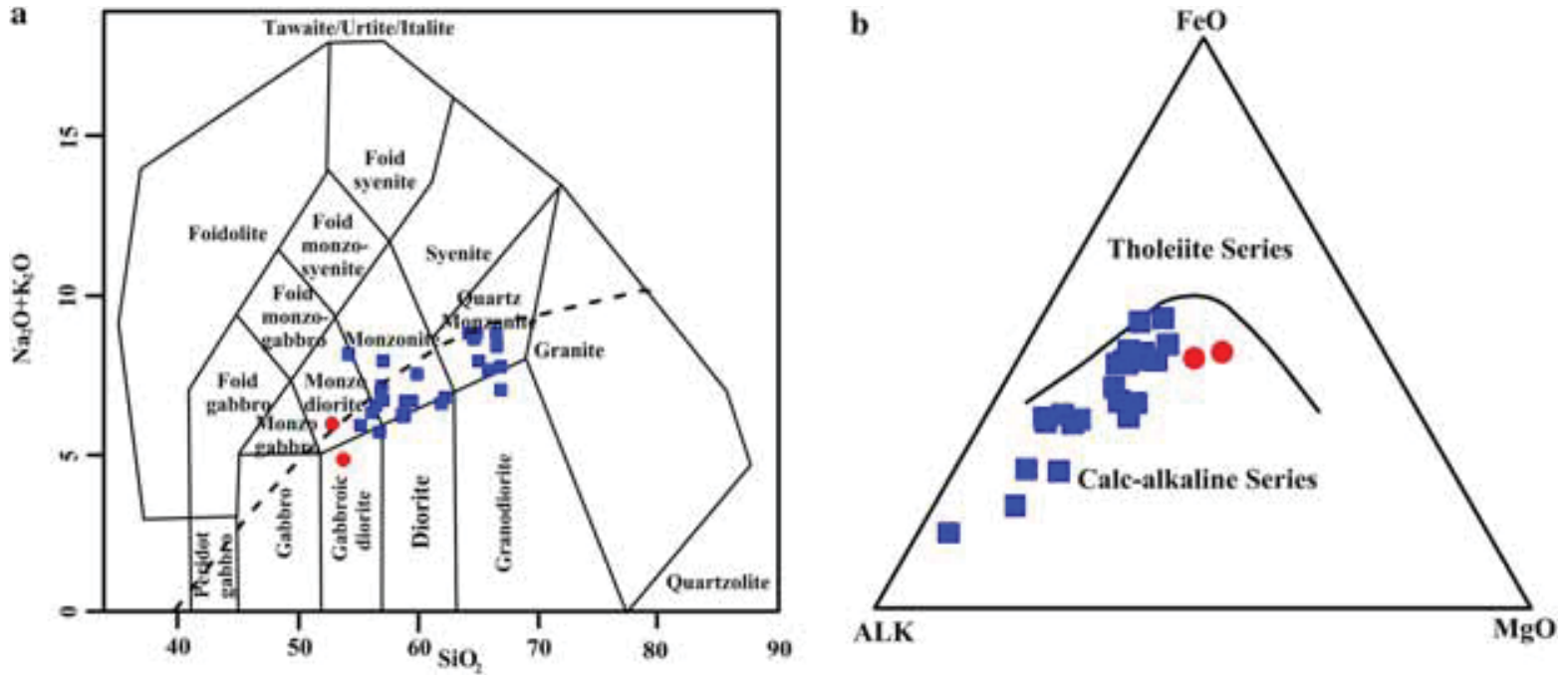

Figure 8. (a) Rock classification diagram (Middlemost 1985) for Chargar intrusions. Samples from Chargar plot into the monzodiorite, monzonite, quartz monzonite, granodiorite and diorite (Type-I) and gabbroic diorite and monzodiorite (Type-II) fields. (b) AFM diagram (Irvine and Baragar 1971) for samples from Chargar intrusions. Blue filled square: southern intrusions (Type-I), red filled circle: western gabbro - gabbro-diorite intrusion (Type-II).
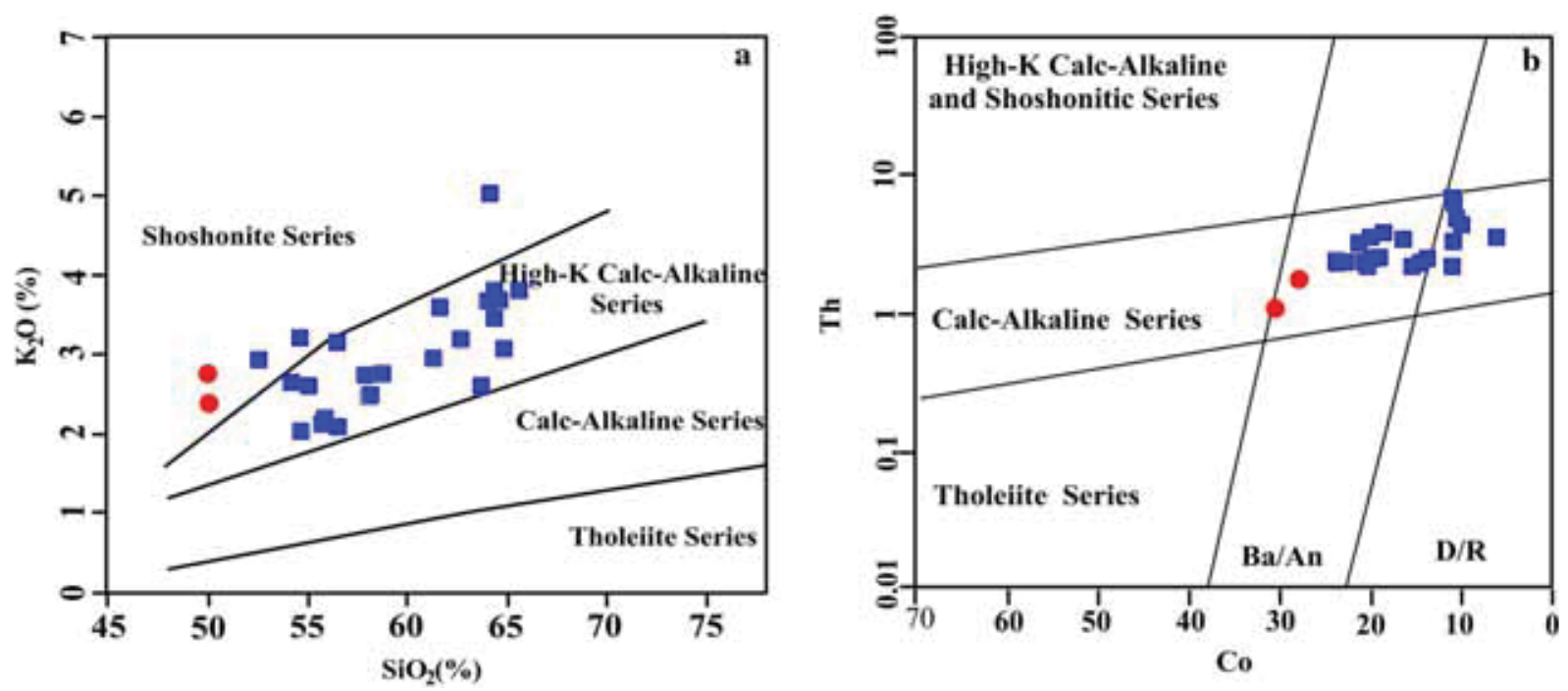

Figure 9. (a) Plot of the Chargar samples on the $\mathrm{K}_{2} \mathrm{O}$ vs. $\mathrm{SiO}_{2}$ diagram (Peccerillo and Taylor 1976). Most of the samples are plotted in the high-K calc-alkaline series field; (b) Plot of Chargar samples on the Th vs. Co classification diagram (Hastie et al. 2007). Blue filled square: southern intrusions (Type-I), red filled circle: western gabbro - gabbro-diorite intrusion (Type-II).

\subsection{Geochemistry}

Major and trace element geochemistry has emerged as a reliable tool to decipher petrogenetic conditions of the rocks. The major and trace element data for the Chargar samples are given in table 1 .

In the Harker diagrams, a distinct gap exists between the two series (figure 7) which can be related to the small number of analyzed samples from the Type-II intrusion and gabbroic rocks from the Type-I intrusion. Major element variations characterize two general types of sources. Each type has a distinct chemical signature representing a separate magmatic evolution. $\mathrm{CaO}, \mathrm{TiO}_{2}, \mathrm{Fe}_{2} \mathrm{O}_{3}$, $\mathrm{MgO}$ and $\mathrm{MnO}$ contents in Type-I decrease with increasing silica content as assumed for FC processes throughout magma evolution in the Chargar region. The $\mathrm{K}_{2} \mathrm{O}$ contents increase with increasing silica contents.

The Chargar intrusive rocks display wide compositional variations. Two groups of rocks were divided based on the existent mineralization, their locations, major and trace elements: Type-I and Type-II. Based on major element geochemistry, the 
Chargar Type-I and Type-II intrusions were classified applying the classification diagram of Middlemost (1985) (figure 8a). This diagram indicates that the southern plutonic rocks (Type-I) plot in the acid to intermediate fields (monzodiorite, monzonite, quartz monzonite, granodiorite, and diorite), while the western plutonic rocks (Type-II) plot in the basic field (gabbroic diorite and monzodiorite) (figure 8a). Intrusive rocks of Chargar area mainly have quartz monzonite composition (figure 8a). The Chargar intrusive rocks are plotted in the sub-alkaline field. The subalkaline series can be separated using AFM triangle diagram into calc-alkaline and tholeiite series (Irvine and Baragar 1971). All samples from the

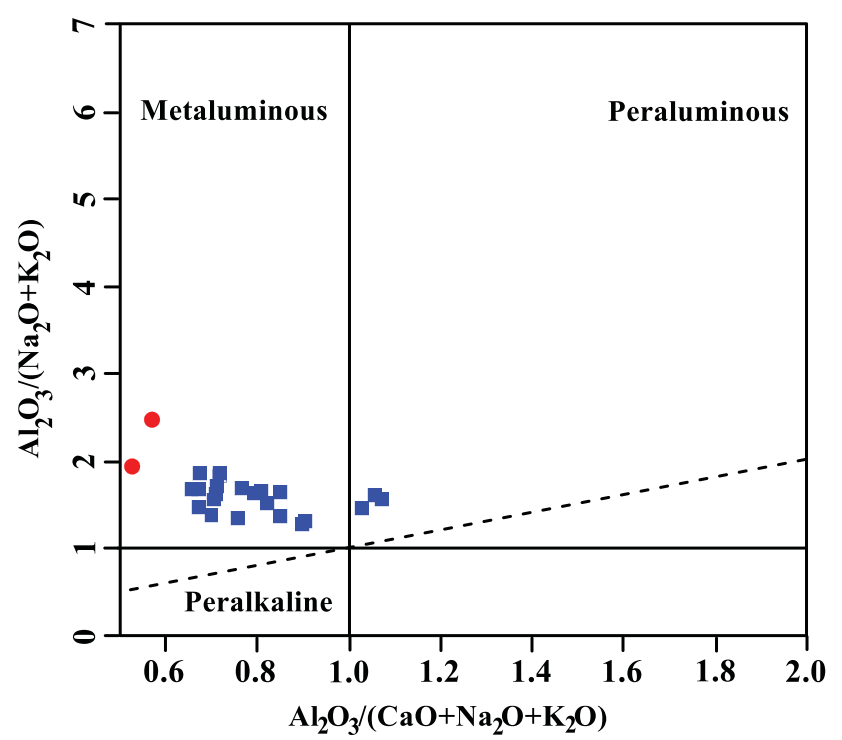

Figure 10. Plot of the Chargar samples on the A/NK vs. A/CNK diagram (Shand 1943) $\left[\mathrm{A} / \mathrm{NK}=\right.$ molar $\mathrm{Al}_{2} \mathrm{O}_{3} /$ $\left(\mathrm{Na}_{2} \mathrm{O}+\mathrm{K}_{2} \mathrm{O}\right) ; \mathrm{A} / \mathrm{CNK}=$ molar $\left.\mathrm{Al}_{2} \mathrm{O}_{3} /\left(\mathrm{CaO}+\mathrm{Na}_{2} \mathrm{O}+\mathrm{K}_{2} \mathrm{O}\right)\right]$. Blue filled square: southern intrusions (Type-I), red filled circle: western gabbro - gabbro-diorite intrusion (Type-II).
Chargar area demonstrate calc-alkaline nature (figure $8 \mathrm{~b}$ ).

The Chargar intrusive rocks have high $\mathrm{K}_{2} \mathrm{O}$ contents so that most of the samples plot in the high- $\mathrm{K}$ calc-alkaline field in a $\mathrm{K}_{2} \mathrm{O}$ vs. $\mathrm{SiO}_{2}$ classification diagram (Peccerillo and Taylor 1976; figure 9a). Based on Th vs. Co diagram (Hastie et al. 2007), all samples plot in the calc-alkaline field (figure $9 \mathrm{~b}$ ). The Chargar intrusive bodies are metaluminous, with the alumina saturation index values $(\mathrm{A} / \mathrm{CNK})$ of $<1$ (figure 10). Usually, the rocks display I-type characteristics involving a positive correlation between $\mathrm{Rb}$ and $\mathrm{Th}$ (White and Chappell 1977; Chappell 1999), which is also confirmed by petrographic studies where hornblende is present as a major mafic phase, that is mostly absent in S-type granitoids.

In the $\mathrm{Zr}$ vs. $\mathrm{SiO}_{2}$ diagram (Collins et al. 1982) and $\mathrm{Na}_{2} \mathrm{O}$ vs. $\mathrm{K}_{2} \mathrm{O}$ diagram (Chappell and White 2001), the Chargar intrusive samples plot in the I-type granite field (figure 11). The hornblende, biotite, and apatite presence; high $\mathrm{CaO}$ and $\mathrm{Na}_{2} \mathrm{O}$ ratios (White and Chappell 1983; Chappell and Stephens 1988; Chappell and White 2001); LILE and LREE enrichment, and HFSE depletion affirm their I-type nature (e.g., Dall'Agnol et al. 2012). Three main mechanisms are proposed for the formation of I-type magmas in subduction zones (e.g., Altherr et al. 2000): (1) partial melting of subducted oceanic crust, (2) metasomatized mantle wedge melting above subducted slab, and (3) partial melting of lower crustal rocks because of the injection of hot mantle magmas.

In the primitive mantle-normalized spider diagrams, all of the Chargar Type-I and Type-II intrusive rocks display LILE (e.g., Cs, Ba, K, and $\mathrm{Pb}$ ) enrichment and negative anomalies of HFSE (e.g., Nb, Zr, and Ti). Depletion of HFSEs implies

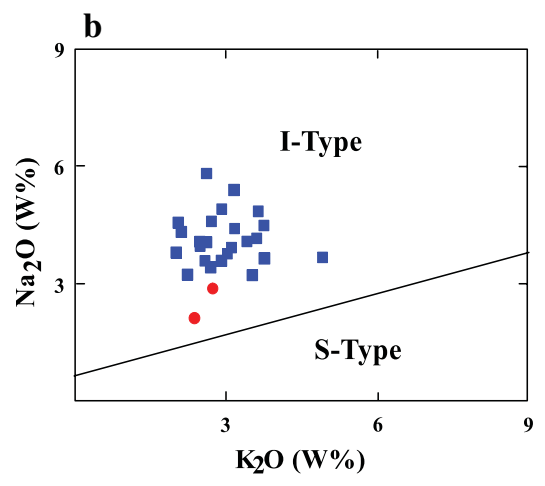

Figure 11. (a) Plot of the Chargar samples on the $\mathrm{Zr}$ vs. $\mathrm{SiO}_{2}$ diagram (Collins et al. 1982); (b) Plot of the Chargar samples on the $\mathrm{Na}_{2} \mathrm{O}$ vs. $\mathrm{K}_{2} \mathrm{O}$ diagram (Chappell and White 2001). Blue filled square: southern intrusions (Type-I), red filled circle: western gabbro - gabbro-diorite intrusion (Type-II). 

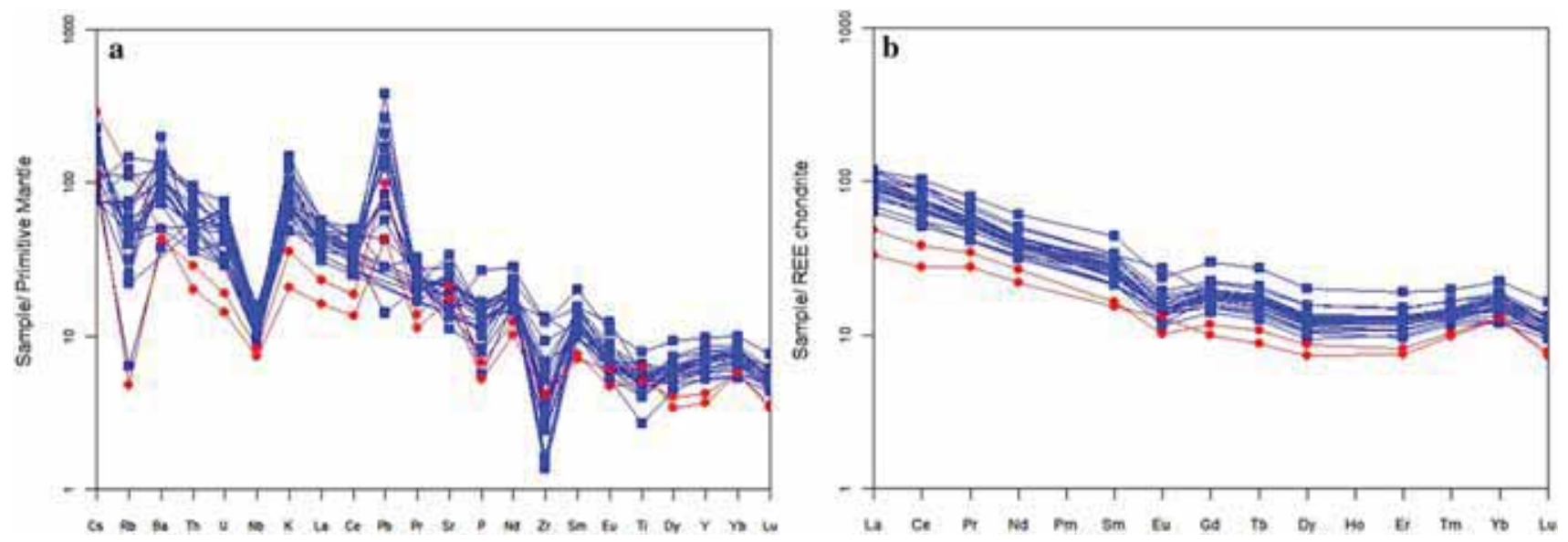

Figure 12. (a) Primitive mantle-normalized (McDonough and Sun 1995) multi-element spider diagram for the Chargar intrusions. (b) Chondrite-normalized (Nakamura 1974) REE patterns for the Chargar intrusions. Blue filled square: southern intrusions (Type-I), red filled circle: western gabbro - gabbro-diorite intrusion (Type-II).

the formation of this suite in a subduction zone setting; location of the HFSEs retain by the subducting slab (Saunders et al. 1980; McDonough 1991). A pronounced negative $\mathrm{Zr}$ anomaly for the Chargar samples is pointing out of a subduction setting (Wang et al. 2013). Characteristics of some gabbroic samples which show weak positive $\mathrm{Eu}$ anomaly in the chondrite-normalized REE pattern and the weak positive $\mathrm{Ti}$ anomaly in the primitive mantle-normalized spider diagram (figure 12a) reflect the abundance of cumulative plagioclase (Bau 1991) and presence of ilmenite or titanomagnetite (Desta et al. 2015).

According to Zheng (2012), positive $\mathrm{Pb}$ anomalies on a primitive mantle-normalized spider diagram (Sun and McDonough 1989) are ascribed to mantle wedge metasomatism in subduction zones by continental crust sediments and also attributed to the continental contamination (Kamber et al. 2002) (figure 12a). Two models for metasomatism are proposed in these zones: a model described by slab-derived fluids and another by hydrous melt. The first has the ability to carry water-soluble elements, not the waterinsoluble elements, but hydrous melts are able to transport both water-soluble and insoluble elements. $\mathrm{Rb} / \mathrm{Y}, \mathrm{Nb} / \mathrm{Y}, \mathrm{Nb} / \mathrm{Zr}$, and $\mathrm{Th} / \mathrm{Zr}$ ratios are used to determine the type of metasomatized media (Kepezhinskas et al. 1997). The samples from the Chargar area indicate high values of $\mathrm{Rb} / \mathrm{Y}$ and $\mathrm{Th} / \mathrm{Zr}$, and lower values of $\mathrm{Nb} / \mathrm{Y}$ and $\mathrm{Nb} / \mathrm{Zr}$, indicating hydrous melt superiority in the parental magma.

Chondrite-normalized REE patterns of the Chargar intrusive rocks show LREE enrichment, flat HREE pattern and relatively high LREE/ HREE ratios (figure 12b). Chondrite-normalized REE patterns show a fractionated trend, variable negative Eu anomalies and flat HREEs which are typical of calc-alkaline magmas. The flat HREE pattern indicates that magma formed at a depth where amphibole was as a stable phase (Rapp and Watson 1995; Barth et al. 2002). Negative Eu anomalies in most of the samples are attributed to plagioclase fractionation, while two gabbroic samples indicate weak positive Eu anomalies which can be related to plagioclase concentration.

\subsection{Tectonic setting}

All of the Chargar intrusions show high LILE enrichment and negative anomalies of $\mathrm{Nb}$ and $\mathrm{Ti}$, mostly along with positive $\mathrm{Pb}$ anomaly which are characteristics commonly found in rocks typical of subduction zone settings (e.g., Hawkesworth et al. 1993; Pearce and Peate 1995). A continental arc is the suggested setting for these rocks based on the primitive mantle-normalized spider diagram where trace element signatures of these rocks show similarity with continental arc samples.

Trace elements are used to separate different tectonic settings of granitoids (e.g., Harris et al. 1986; Pearce 1996). Based on the $\mathrm{Yb} / \mathrm{Ta}$ vs. Rb diagram (Pearce 1996), all of Chargar granitoid samples fall into the volcanic arc granitoid (VAG) field (figure 13a). On the Ta vs. Th and Th/Yb vs. $\mathrm{Ta} / \mathrm{Yb}$ tectonic setting discrimination diagrams (Gorton and Schandl 2000), most Chargar granitoids plot in the field of active continental margin (figure 13b, c). Based on Zr vs. Y diagram (Muller 

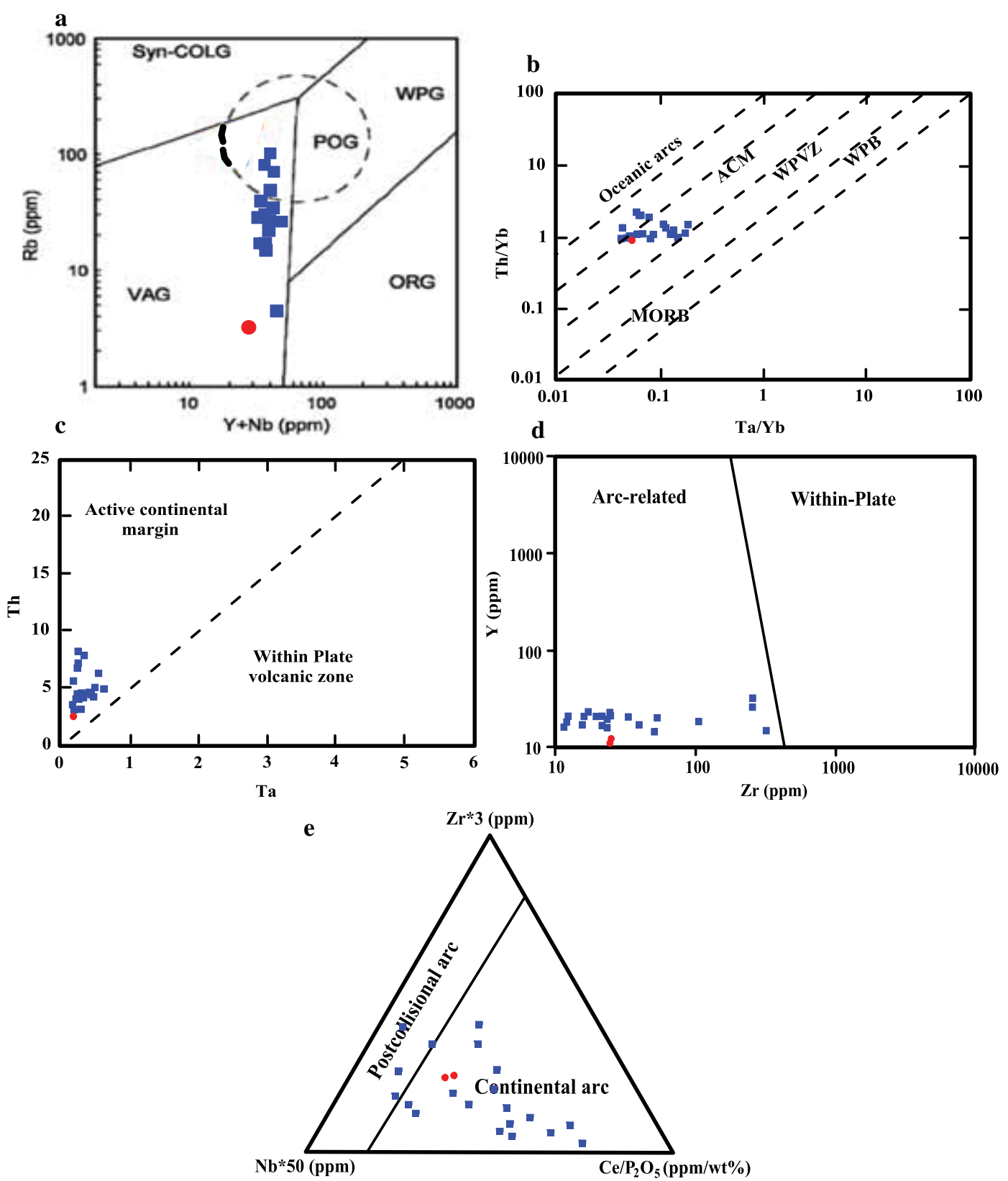

Figure 13. Tectonic setting discrimination diagrams of Chargar intrusive rocks. (a) Rb vs. Y+Nb tectonic setting discrimination diagram (Pearce 1996) with fields of volcanic arc (VAG), syn-collisional (syn-COLG), within-plate (WPG), and oceanic ridge (ORG) granites. Samples from the Chargar granitoids plot in the VAG field; (b) Th/Yb vs. Ta/Yb diagram (Gorton and Schandl 2000). OA = Oceanic Arcs, ACM= Active Continental Margins, WPVZ= Within-Plate Volcanic Zones, WPB= Within-Plate Basalts, MORB= Mid-Ocean Ridge Basalts; (c) Th vs. Ta diagram (Gorton and Schandl 2000); (d) Y vs. Zr plot (Muller and Groves 1997); (e) $\mathrm{Zr} \times 3-\mathrm{Nb} \times 50-\mathrm{Ce} / \mathrm{P}_{2} \mathrm{O}_{5}$ triangular diagram (Muller and Groves 1997). Blue filled square: southern intrusions (Type-I), red filled circle: western gabbro - gabbro-diorite intrusion (Type-II).

and Groves 1997), Chargar granitoids plot in the field of arc or subduction-related suites (figure 13d). Furthermore, on the $\mathrm{Zr} * 3-\mathrm{Nb} * 50-\mathrm{Ce} / \mathrm{P}_{2} \mathrm{O}_{5}$ triangular diagram (Muller and Groves 1997), most Chargar samples plot in the continental arc setting and a few samples plot on post-collisional arc setting (figure 13e). In general, it can be concluded that all samples are related to subduction calc-alkaline magmatism.

\section{Discussion}

Geochemical variations in magmatic rocks can determine magmatic processes such as fractional crystallization of the parental magma. In the $\mathrm{Ba}-\mathrm{Sr}$ diagram (Arslan and Aslan 2006), the Chargar intrusions show non-linear trends which imply modifications in the fractionating assemblage of more complex processes. However, the general 


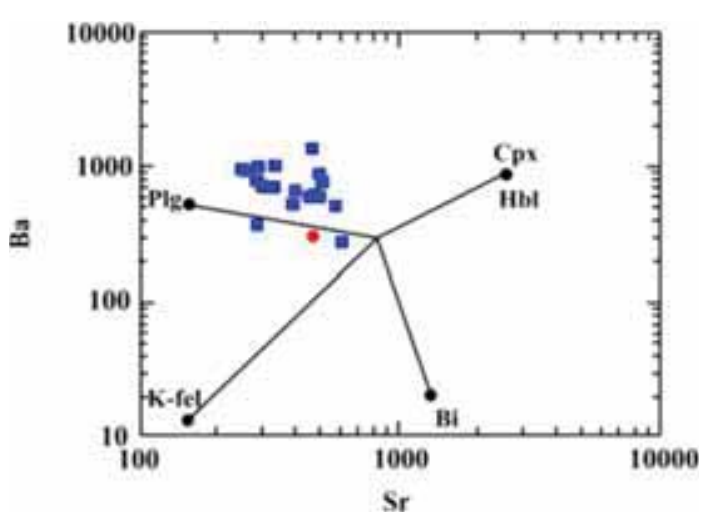

Figure 14. Ba vs. Sr diagram (Arslan and Aslan 2006) and plots for the Chargar intrusions. The vectors indicate the influence of fractionation of plagioclase (Plg), potassium feldspar (K-fel), biotite (Bi) and hornblende ( $\mathrm{Hbl}$ ) on the composition of the residual liquids. Blue filled square: southern intrusions (Type-I), red filled circle: western gabbro - gabbrodiorite intrusion (Type-II).

trend can indicate plagioclase fractionation (figure 14).

The geochemical characteristics of the Chargar intrusions suggest that these are derived by partial melting from a metasomatized sub-continental lithospheric mantle source in a subduction zone. The geochemical features of some samples imply their shoshonitic affinity and emphasize a postcollisional zone (e.g., Liégeois et al. 1998). They also can be produced from an active subduction zone (Blatter et al. 2001; Bonev and Beccaletto 2007), metasomatized sub-continental lithospheric mantle (Aldanmaz et al. 2000; Seghedi et al. 2004), or assimilation of crustal rocks (Meen 1987; Feeley and Cosca 2003).

$\mathrm{Ba} / \mathrm{La}$ vs. Th/Yb diagram (Hou et al. 2012) for the Chargar intrusions indicates the addition of the fluids from the subducted slab into their source region and slab-derived enrichment (figure 15). In this diagram, the Chargar Type-I samples reveal elevated $\mathrm{Th} / \mathrm{Yb}$ with relatively narrow $\mathrm{Ba} / \mathrm{La}$ variation. Usually, magmatic rocks derived from a metasomatized mantle source by a fluid component are reasonable to have high $\mathrm{Ba}$ and $\mathrm{Pb}$ concentrations and high $\mathrm{Ba} / \mathrm{La}$ and $\mathrm{Ba} / \mathrm{Th}$ ratios, but magmas with a strong imprint of sediment-derived melts in their source region should have high $\mathrm{Th}$ contents and high $\mathrm{Th} / \mathrm{Yb}$ and $\mathrm{Nb} / \mathrm{Y}$ ratios (Hawkesworth et al. 1997; Woodhead et al. 2001; Kelemen et al. 2003; Zhao et al. 2009; Guo et al. 2013, 2015; Liu et al. 2014). Thus, the high Th/Yb ratios for the samples indicate the metasomatized mantle source for the Chargar intrusions by sediment-derived melts (figure 15). In contrast, the

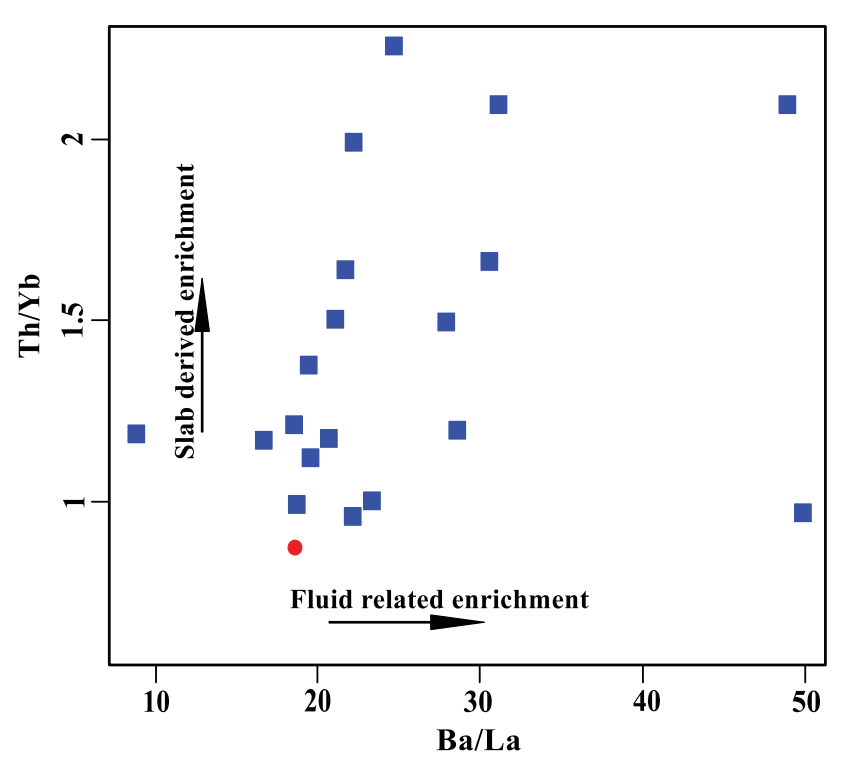

Figure 15. Ba/La vs. Th/Yb diagram (modified after Hou et al. 2012) for the Chargar intrusions. Blue filled square: southern intrusions (Type-I), red filled circle: western gabbro gabbro-diorite intrusion (Type-II).

high $\mathrm{Ba}$ and $\mathrm{Pb}$ contents, and high $\mathrm{Ba} / \mathrm{La}$ ratios are related to an enriched mantle source by fluids.

The high $(\mathrm{La} / \mathrm{Sm})_{N}(\sim>3.5)$ and low $(\mathrm{Tb} / \mathrm{Yb})_{N}$ values for Chargar intrusions suggest their possible generation by partial melting of a mantle source with amphibole-bearing spinel peridotite composition (figure 16). Incompatible element ratios like La/Sm and $\mathrm{Sm} / \mathrm{Yb}$ are influenced by partial melting and fractional crystallization processes. When partial melting is lower, La/Sm would be high. In general, high $\mathrm{La} / \mathrm{Yb}$ ratios indicate smaller melt fractions and/or garnet as the main residual phase and low $\mathrm{La} / \mathrm{Yb}$ ratios show a dominated melting regime by larger melt fraction and/or spinel as the predominant residual phase (Yang et al. 2007).

The relationship between the Chargar intrusions and their mineralogy and geochemistry suggest that they have differentiated from a common parent magma. It seems that the parent magma of the Chargar intrusions was basic in composition, and the gabbros represent an accumulation of earlyformed crystals from this magma. This accumulation of early-formed crystals was separated from the remaining liquid by pressure due to crustal movements, and this liquid was injected into higher levels in the crust and formed the gabbro, pyroxene quartz monzodiorite, quartz monzodiorite, and the quartz syenite, respectively. The concept of the origin for the quartz syenite as the product of crystallization of a magmatic liquid from which the products of early crystallization had been removed is supported by certain criteria developed by Bowen (1937). 


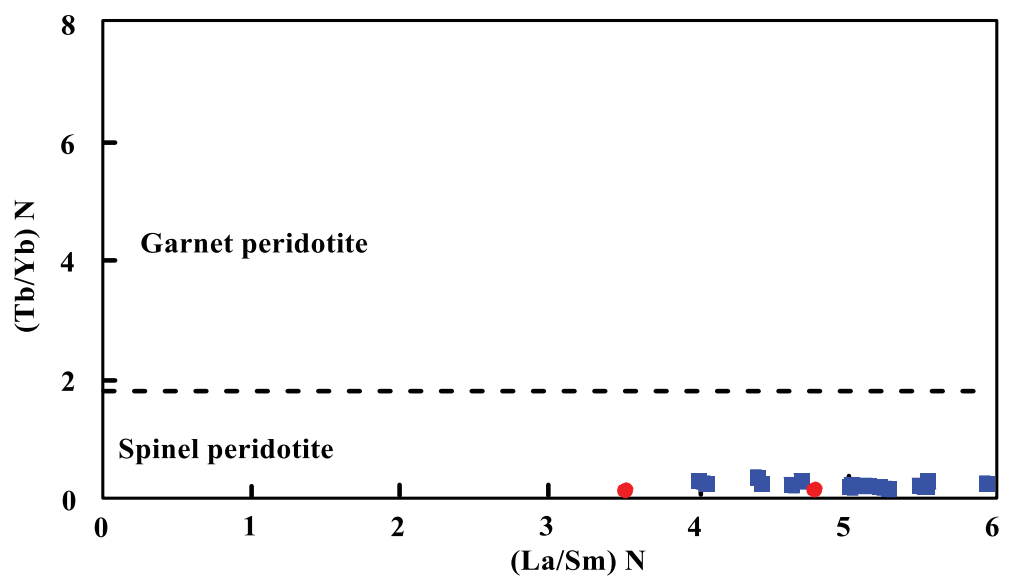

Figure 16. $(\mathrm{La} / \mathrm{Sm})_{N}$ vs. $(\mathrm{Tb} / \mathrm{Yb})_{N}$ diagram (Wang et al. 2002) for the Chargar intrusions. The horizontal dashed line separates the fields of garnet-bearing peridotite and spinel-bearing peridotite; Blue filled square: southern intrusions (Type-I), red filled circle: western gabbro - gabbro-diorite intrusion (Type-II).

Positive Eu anomaly corresponds to plagioclaserich gabbroic rocks. It seems that the gabbroic intrusions could be the cumulative parts of the magma chamber for the Chargar intrusions. Such cumulative assemblages imply post-collisional transtensional movements (Desta et al. 2015). Furthermore, the positive Eu anomaly is attributed to the mixing of a mantle component with crustal melts (Mazzucchelli et al. 1992). It is suggested that the primary magma for the Chargar gabbro - gabbro-diorite (Type-II) should be derived from the lithospheric mantle intensively modified by continental crust.

\section{Conclusions}

Two different groups of rocks were separated in the Chargar area of the Tarom belt in Alborz, based on mineralization occurrence as well as major, trace and REE geochemistry.

The Type-I rocks are composed of gabbromonzodiorite-quartz syenite compositions. They are calc-alkaline and metaluminous and in the diagram of the major elements a negative correlation of $\mathrm{SiO}_{2}$ relative to $\mathrm{Al}_{2} \mathrm{O}_{3}, \mathrm{Fe}_{2} \mathrm{O}_{3}, \mathrm{MgO}, \mathrm{CaO}$ and $\mathrm{TiO}_{2}$ and a positive correlation with $\mathrm{K}_{2} \mathrm{O}$. Type-I rocks are enriched in LREE against HREE. In the primitive mantle-normalized spider diagram, negative $\mathrm{Ti}, \mathrm{Nb}$, and $\mathrm{Zr}$ anomalies and a mostly positive anomaly of $\mathrm{Pb}$ can be seen. The LIL elements $\mathrm{Cs}$ and $\mathrm{Ba}$ are enriched relative to HFSE. The crustal assimilation/contamination together with fractional crystallization was an important process. Geochemical signatures have a similar appearance to continental arc setting.
The Type-II rocks are mafic metaluminous. TypeII rocks were possibly generated at the beginning of the development of the arc setting or it could have been related to small extensions in the continental arc environment. The sample with the lowest $\mathrm{SiO}_{2}$ has moderate $\mathrm{MgO}$ content $(5.13 \%)$. These attributes suggest their derivation, not from a primary magma, but experiencing fractional crystallization probably associated with crustal assimilation. The crustal assimilation as an important process changed the original magma composition. Affected rocks by intense partial melting will show special evidence for this melt extraction. Strong evidence for the melt extraction and fractionation of basaltic magmas in the lower crust would be a positive Eu anomaly (Rudnick 1992). Therefore, intrusive bodies in the Chargar area were caused by the subduction of the Arabian plate beneath the central Iran plate and their subsequent collision.

\section{Acknowledgements}

This paper is a part of the first author's $\mathrm{PhD}$ thesis at Tarbiat Modares University, Tehran, Iran. An anonymous reviewer is thanked for the constructive review of the manuscript. Dr Rajneesh Bhutani is appreciated for careful editorial handling of the manuscript.

\section{References}

Aldanmaz E, Pearce J A, Thirlwall M F and Mitchell J G 2000 Petrogenetic evolution of late Cenozoic, post-collision volcanism in western Anatolia, Turkey; J. Volcanol. Geotherm. Res. 102 67-95. 
Altherr R, Holl A, Ernst H and Kreuzer H 2000 Highpotassium, calc-alkaline I-type plutonism in the European Variscides: Northern Vosges (France) and northern Schwarzwald (Germany); Lithos 50(1) 51-73, https://doi.org/ 10.1016/S0024-4937(99)00052-3.

Arslan M and Aslan Z 2006 Mineralogy, petrography and whole-rock geochemistry of the Tertiary granitic intrusions in the eastern Pontides, Turkey; J. Asian Earth Sci. 27(2) 177-193, https://doi.org/10.1016/j.jseaes.2005.03.00.

Azizi H, Mehrabi B and Akbarpour A 2009 Genesis of Tertiary magnetite-apatite deposits, southeast of Zanjan, Iran; Res. Geol. 59 330-341.

Barth M G, Foley S F and Horn I 2002 Partial melting in Archean subduction zones: Constraints from experimentally determined trace element partition coefficients between eclogitic minerals and tonalitic melts under upper mantle conditions; Precamb. Res. 113 323-340, https:// doi.org/10.1016/s0301-9268(01)00216-9.

Bau M 1991 Rare-earth element mobility during hydrothermal and metamorphic fluid-rock interaction and the significance of the oxidation state of europium. Chem. Geol. 93 (3-4) 219-230.

Blatter D L, Carmichael I S E, Deino A L and Renne P R 2001 Neogene volcanism at the front of the central Mexican volcanic belt: Basaltic andesites to dacites, with contemporaneous shoshonites and high-TiO 2 lava; Geol. Soc. Am. Bull. 113 1324-1342, https://doi.org/10.1130/00167606(2001)113<1324:NVATFO >2.0.CO;2.

Bonev N and Beccaletto L 2007 From syn- to post-orogenic Tertiary extension in the north Aegean region: Constraints on the kinematics in the eastern Rhodope-Thrace, Bulgaria-Greece and the Biga Peninsula, NW Turkey; In: The geodynamics of the Aegean and Anatolia (eds) Taymaz T et al., Geol. Soc. London, Spec. Publ. 291 113-114.

Bowen N L 1937 Recent high-temperature research on silicates and its significance in igneous geology; Am. J. Sci. 233 $1-21$.

Brunet M F, Korotaev M V, Ershov A V and Nikishin A M 2003 The South Caspian basin: A review of its evolution from subsidence modelling; Sedim. Geol. 156 119-148, https://doi.org/10.1016/s0037-0738(02)00285-3.

Castro A, Aghazadeh M, Badrzadeh Z and Chichorro M 2013 Late Eocene-Oligocene post-collisional monzonitic intrusions from the Alborz magmatic belt, NW Iran: An example of monzonite magma generation from a metasomatized mantle source; Lithos 180-181 109-127.

Chappell B W 1999 Aluminium saturation in I- and S-type granites and the characterization of fractionated haplo granites; Lithos 46 535-551, https://doi.org/10.1016/ s0024-4937(98)00086-3.

Chappell B W and Stephens W E 1988 Origin of infra crustal (I-type) granite magmas; In: The origin of granites, Earth Env. Sci. Trans. Roy. Soc. 79(2-3) 71-86, Turkey; In: Comptes rendus des Séance de la Société de Physique et d'Histoire Naturelle de Genève 10 141-150.

Chappell B W and White A J R 2001 Two contrasting granite types: 25 years later; Austr. J. Earth Sci. 48(4) 489-499, https://doi.org/10.1046/j.1440-0952.2001.00882.x.

Collins W J, Beams S D, White A J R and Chappell B W 1982 Nature and origin of A-type granites with particular reference to southeastern Australia; Contrib. Mineral. Petrol. 80 189-200.
Dall'Agnol R, Frost C D and Ramo T 2012 IGCP Project 510 A-type granites and related rocks through time: Project vita, results and contribution to granite research; Lithos $1511-16$.

Desta M T, Ishiwatari A, Machi S, Arai Sh, Tamura A, Ledneva G V, Sokolov S D, Moiseev A V and Bazylev B A 2015 Petrogenesis of Triassic gabbroic and basaltic rocks from Chukotka, NE Russia: Eastern end of the 'arc-type' Siberian LIP? J. Miner. Petrol. Sci. 110 249-275.

Esmaeli M, Lotfi M and Nezafati N 2015 Fluid inclusion and stable isotope study of Khalyfehlou copper deposit, southeast Zanjan, Iran; Arab. J. Geosci. 8 9625-9633.

Feeley T C and Cosca M A 2003 Time vs. composition trends of magmatism at Sunlight volcano, Absaroka volcanic province, Wyoming; Geol. Soc. Am. Bull. 115 714-772, https://doi.org/10.1130/0016-7606(2003)115<0714:TVCT $\mathrm{OM}>2.0 . \mathrm{CO} ; 2$.

Gill J B 1981 Orogenic Andesites and Plate Tectonics; Springer, Berlin, 390p.

Gorton M P and Schandl E S 2000 From continents to island arcs: A geochemical index of tectonic setting for arc-related and within-plate felsic to intermediate volcanic rocks; Can. Mineral. 38 1065-1073.

Guest B, Axen G J, Lam P S and Hassanzadeh J 2006 Late Cenozoic shortening in the west-central Alborz Mountains, northern Iran, by combined conjugate strike-slip and thinskinned deformation; Geosphere 2 35-52, https://doi.org/ 10.1130/ges00019.1.

Guest B, Horton B K, Axen G J, Hassanzadeh J and McIntosh W C 2007 Middle to late Cenozoic basin evolution in the western Alborz Mountains: Implications for the onset of collisional deformation in northern Iran; Tectonics $\mathbf{2 6}$ TC6011, https://doi.org/10.1029/2006tc002091.

Guo Z F, Wilson M, Zhang M, Cheng Z and Zhang L 2013 Postcollisional, K-rich mafic magmatism in south Tibet: Constraints on Indian slab-to-wedge transport processes and plateau uplift; Contrib. Mineral. Petrol. 165 1311-1340.

Guo Z F, Wilson M, Zhang M, Cheng Z and Zhang L 2015 Post-collisional ultrapotassic mafic magmatism in south Tibet: Products of partial melting of pyroxenite in the mantle wedge induced by roll-back and delamination of the sub-ducted Indian continental lithosphere slab; J. Petrol. 56 1365-1406.

Harris N B W, Pearce J A and Tindle A G 1986 Geochemical characteristics of collision-zone magmatism; In: Collision Tectonics (eds) M P Coward and A C Ries, Geol. Soc. London, Spec. Publ., pp. 67-81.

Hassanzadeh J, Axen G J, Guest B, Stockli D F and Ghazi A M 2004 The Alborz and NW Urumieh-Dokhtar magmatic belts, Iran: Rifted parts of a single ancestral arc; Geol. Soc. Am. Abstr. Programs 36(5) 434.

Hastie A R, Kerr A C, Pearce J A and Mitchell S F 2007 Classification of altered volcanic island arc rocks using immobile trace elements: Development of the Co-Th discrimination diagram; J. Petrol. 48 2341-2357.

Hawkesworth C J, Gallagher K, Hergt J M and McDermott F 1993 Mantle and slab contributions in arc magmas; Ann. Rev. Earth Planet. Sci. 2 175-204.

Hawkesworth C, Turner S, Peate D, McDermott F and van Calsteren P 1997 Elemental U and Th variations in island arc rocks: Implications for U-series isotopes; Chel. Geol. 139 207-221. 
Hou T, Zhang Z C, Encarnacion J and Santosh M 2012 Petrogenesis and metallogenesis of the Taihe gabbroic intrusion associated with Fe-Ti-oxide ores in the Panxi district, Emeishan large igneous province, southwest China; Ore Geol. Rev. 49 109-127.

Hirayama K, Samimi M, Zahedi M and Hushmand-Zadeh A 1966 Geology of Taroum district, western part (Zanjan area north-west Iran), Geological Survey of Iran.

Hirayama K, Haghipour A and Hajian J 1965 Geology of the Zanjan area: The Tarom district, eastern part (Zanjan area, northwest Iran), with 1:100,000 map; Geological Survey of Iran, Tehran 28 33p.

Hosseini M, Moosavi E and Rasouli Jamadi F 2017 Abhar 1:100,000 geological map; Geological Survey of Iran.

Irannezhadi M R, Ghorbani M R, Vossoughi M and Pourmoafi M 2007 Tertiary arc related volcanism in Central Alborz Mountains; Geophys. Res. Abs. 9867.

Irvine T N and Baragar W R A 1971 A guide to the chemical classification of the common volcanic rocks; Can. J. Earth Sci. 8 523-548.

Kamber B S, Ewart A, Collerson K D, Bruce M C and McDonald G D 2002 Fluid-mobile trace element constraints on the role of slab melting and implications for Archaean crustal growth models; Contrib. Mineral. Petrol. 144 $38-56$.

Kelemen P B, Hanghøj K and Greene A 2003 Subductionrelated magmatic arcs, with an emphasis on primitive andesite and lower crust; In: The Crust (eds) Holland H D and Turekian K K, V. 3. Treatise on Geochemistry, Oxford, UK: Elsevier-Pergamon, pp. 593-659.

Kepezhinskas P, McDermott F, Defant M J, Hochstaedter A, Drummond M S, Hawkesworth C J, Koloskov A, Maury R $\mathrm{C}$ and Bellon H 1997 Trace element and $\mathrm{Sr}-\mathrm{Nd}-\mathrm{Pb}$ isotopic constraints on a three-component model of Kamchatka Arc petrogenesis; Geochim. Cosmochim. Acta 61 577-600, https://doi.org/10.1016/s0016-7037(96)00349-3.

Kouhestani H, Azimzadeh A M, Mokhtari M A A and Ebrahimi M 2017 Mineralization and fluid evolution of epithermal base metal veins from the Aqkand deposit, NW Iran; N. Jb. Miner. Abh. (J. Min. Geochem.) 194(2) 139-155.

Kouhestani H, Mokhtari M A A, Chang Z and Johnson C A 2018 Intermediate sulfidation type base metal mineralization at Aliabad-Khanchy, Tarom-Hashtjin metallogenic belt, NW Iran; Ore Geol. Rev. 93 1-18.

Liégeois J P, Navez J, Hertogen J and Black R 1998 Contrasting origin of post-collisional high-K calc-alkaline and shoshonitic versus alkaline and peralkaline granitoids. The use of sliding normalization; Lithos 45 1-28, https:// doi.org/10.1016/s0024-4937(98)00023-1.

Liu D, Zhao Z, Zhu D C, Niu Y, DePaolo D J, Mark Harrison T, Mo X, Dong G, Zhou S, Sun C, Zhang Z and Liu J 2014 Post-collisional potassic and ultrapotassic rocks in southern Tibet: Mantle and crustal origins in response to India-Asia collision and convergence; Geochim. Cosmochim. Acta 143 $207-231$.

Mazzucchelli M, Rivalenti G, Vannucci R, Bottazzi P, Ottolini L, Hofmann A and Parenti M 1992 Primary positive Eu anomaly in clinopyroxenes of low-crust gabbroic rocks; Geochim. Cosmochim. Acta 56(6) 2363-2370.

McDonough W F 1991 Partial melting of subducted oceanic crust and isolation of its residual eclogitic lithology; Phil. Trans. Roy. Soc. London, Ser. A 335 407-418.
McDonough W F and Sun S S 1995 The composition of the Earth; Chem. Geol. 120 223-253.

Meen J K 1987 Formation of shoshonites from calc-alkaline basalt magmas: Geochemical and experimental constraints from the type locality; Contrib. Mineral. Petrol. 97 333-351, https://doi.org/10.1007/bf00371997.

Mehrabi B, Ghasemi Siani M, Goldfarb R, Azizi H, Ganerod M and Marsh E E 2016 Mineral assemblages, fluid evolution and genesis of polymetallic epithermal veins, Gulojeh district, NW Iran; Ore Geol. Rev. 78 41-57.

Middlemost E A K 1985 Magmas and magmatic rocks: An introduction to igneous petrology; Longman Group, UK, pp. 73-87.

Morley C K, Kongwung B, Julapour A A, Abdolghafourian M, Hajian M, Waples D, Warren J, Otterdoom H, Srisuriyon K and Kazemi H 2009 Structural development of a major late Cenozoic basin and transpressional belt in central Iran: The central basin in the Qom-Saveh area; Geosphere 5 1-38.

Mousavi Motlagh S H and Ghaderi M 2019 The Chargar $\mathrm{Au}-\mathrm{Cu}$ deposit: An example of low-sulfidation epithermal mineralization from the Tarom subzone, NW Iran; $N$. Jb. Miner. Abh. (J. Min. Geochem.), 196(1) 43-66, https:// doi.org/10.1127/njma/2019/0158.

Muller D and Groves D I 1997 Potassic igneous rocks and associated Gold-Copper mineralization; Springer-Verlag, Berlin, Heidelberg, 238p.

Nabatian G, Giang S Y, Honarmand M and Neubauer F 2016 Zircon $\mathrm{U}-\mathrm{Pb}$ ages, geochemical and $\mathrm{Sr}-\mathrm{Nd}-\mathrm{Pb}-\mathrm{Hf}$ isotopic constraints on petrogenesis of the Tarom-Olya pluton, Alborz magmatic belt; Lithos 244 43-58, https://doi.org/ 10.1016/j.lithos.2015.11.020.

Nabatian G, Ghaderi M, Neubauer F, Honarmand M, Lui X, Dong Y, Jiang S-Y and Bernroider M 2014 Petrogenesis of Tarom high-potassic granitoids in the Alborz-Azarbaijan belt, Iran: Geochemical, U-Pb zircon and $\mathrm{Sr}-\mathrm{Nd}-\mathrm{Pb}$ isotopic constraints; Lithos 184-187 324-345.

Nakamura N 1974 Determination of REE, Ba, Mg, Na and K in carbonaceous and ordinary chondrites; Geochim. Cosmochim. Acta 38 757-775.

Pearce J 1996 Sources and settings of granitic rocks; Episodes 19 120-125.

Pearce J A and Peate D W 1995 Tectonic implications of the composition of volcanic arc magmas; Ann. Rev. Earth Planet. Sci. 23 251-285.

Peccerillo A and Taylor S R 1976 Geochemistry of Eocene calc-alkaline volcanic rocks from Kastamonu area, northern Turkey; Contrib. Mineral. Petrol. 58 63-81.

Rapp R P and Watson E B 1995 Dehydration melting of metabasalt at 8-32 kbar: Implications for continental growth and crust-mantle recycling; J. Petrol. 36 891-931, https://doi.org/10.1093/petrology/36.4.891.

Rudnick R L 1992 Restites, Eu anomalies, and the lower continental crust; Geochim. Cosmochim. Acta 56 963-970.

Seghedi I, Downes H, Szakács A, Mason P R D, Thirl-wall M F, Roşu E, Pécskay Z, Márton E and Panaiotu C 2004 Neogene-Quaternary magmatism and geodynamics in the Carpathian-Pannonian region; A synthesis: Lithos $\mathbf{7 2}$ 117-146.

Shand S J 1943 Eruptive Rocks; 2nd edn, John Wiley, New York.

Sun S S and McDonough W F 1989 Chemical and isotopic systematics of oceanic basalts: Implications for mantle 
composition and processes; In: Magmatism in the Ocean Basins (eds) Saunders A D and Norry M J, Geol. Soc. London, Spec. Publ. 42 313-435.

Saunders J C, Dolgin K G and Lowry L D 1980 The maturation of frequency selectivity in C57BL/6J mice studied with auditory evoked response tuning curves; Brain Res. 187 69-79.

Verdel Ch, Wernicke B P, Hassanzadeh J and Guest B 2011 A Paleogene extensional arc flare-up in Iran; Tectonics $\mathbf{3 0}$ TC3008, https://doi.org/10.1029/2010tc002809.

Vincent S J, Allen M B, Ismail-Zadeh A D, Flecker R, Foland K A and Simmons M D 2005 Insights from the Talysh of Azerbaijan into the Paleogene evolution of the South Caspian region; Geol. Soc. Am. Bull. 117 1513-1533, https://doi.org/10.1130/b25690.1.

Wang K, Plank T, Walker J D and Smith E I 2002 A mantle melting profile across the basin and range, SWUSA; J. Geophys. Res. 107, ECV 5-1-ECV, 5-21, https://doi. org/10.1029/2001JB000209.

Wang H, Wu Y B, Qin Z W, Zhu L Q, Liu Q, Liu X C, Gao S, Wijbrans J R, Zhou L, Gong H J and Yuan H L 2013 Age and geochemistry of Silurian gabbroic rocks in the Tongbai orogen, central China: Implications for the geodynamic evolution of the North Qinling arc-back-arc system; Lithos 179 1-15.

White A J R and Chappell B W 1977 Ultrametamorphism and granitoid genesis; Tectonophys. 43 7-22, https://doi.org/ 10.1016/0040-1951(77)90003-8.

Corresponding editor: RajneEsh BhUtani
White A J R and Chappell B W 1983 Granitoid types and their distribution in the Lachlan Fold Belt, southeastern Australia; Geol. Soc. Am. Memoir 159 21-34.

Whitney D and Evans B M 2010 Abbreviations for names of rock-forming minerals; Am. Mineral. 95 185-187.

Woodhead J D, Hergt J M, Davidson J P and Eggins S M 2001 Hafnium isotope evidence for 'conservative' element mobility during subduction zone processes; Earth Planet. Sci. Lett. 192 331-346.

Yang J H, Sun J F, Chen F K, Wilde S A and Wu F Y 2007 Sources and petrogenesis of late Triassic dolerite dikes in the Liaodong peninsula: Implications for post-collisional lithosphere thinning of the eastern North China Craton; J. Petrol. 48 1973-1997.

Yasami N, Ghaderi M, Mokhtari M A A and Mousavi Motlagh S H 2018 Petrogenesis of the two phases of intrusive rocks at Chodarchay, NW Iran: Using trace and rare earth elements, NW Iran; Arab. J. Geosci. 11(20) 605, https:// doi.org/10.1007/s12517-018-3942-3.

Zhao Z, Mo X, Dilek Y, Niu Y, De Paolo D J, Robinson P, Zhu D, Sun C, Dong G and Zhou S 2009 Geochemical and $\mathrm{Sr}-\mathrm{Nd}-\mathrm{Pb}-\mathrm{O}$ isotopic compositions of the post-collisional ultrapotassic magmatism in SW Tibet: Petrogenesis and implications for India intra-continental subduction beneath southern Tibet; Lithos 113 190-212.

Zheng Y F 2012 Metamorphic chemical geodynamics in continental subduction zones; Chem. Geol. 328 5-48. 